\title{
Reflectionless Solutions for Square Matrix NLS with Vanishing Boundary Conditions
}

\author{
Francesco Demontis*, Cornelis van der Mee*
}

\begin{abstract}
In this article we derive the reflectionless solutions of the $2+2$ matrix NLS equation with vanishing boundary conditions and four different symmetries by using the matrix triplet method of representing the Marchenko integral kernel in separated form. Apart from using the Marchenko method, these solutions are also verified by direct substitution in the $2+2$ NLS equation.
\end{abstract}

\section{Introduction}

In this paper we study the matrix nonlinear Schrödinger equation

$$
i Q_{t}+Q_{x x}-2 Q R Q=0_{2 \times 2}
$$

where $Q(x, t)$ is a $2 \times 2$ complex matrix valued function and $R=\Sigma Q^{\dagger} \Omega$ for fixed matrices $\Sigma, \Omega \in\left\{ \pm I_{2}, \pm \sigma_{3}\right\}$. Here the dagger indicates the complex conjugate transpose and $\sigma_{1}=\left(\begin{array}{ll}0 & 1 \\ 1 & 0\end{array}\right), \sigma_{2}=\left(\begin{array}{cc}0 & -i \\ i & 0\end{array}\right)$, and $\sigma_{3}=\left(\begin{array}{cc}1 & 0 \\ 0 & -1\end{array}\right)$ are the Pauli matrices. These choices of $\Sigma$ and $\Omega$ lead to four physically relevant cases (see [22] for more details on this fact): case 1 (the defocusing case): $\Sigma=\Omega=I_{2}$, case 2 (the focusing case): $\Sigma=-\Omega=I_{2}$, case $3: \Sigma=\Omega=\sigma_{3}$, and case 4: $\Sigma=-\Omega=\sigma_{3}$, where we note that a simultaneous sign change of $\Sigma$ and $\Omega$ does not create new cases. In addition to these four symmetries, we also discuss the special case where $Q(x, t)$ is a complex symmetric matrix.

*Dipartimento di Matematica e Informatica, Università di Cagliari, Viale Merello 92, 09123 Cagliari, Italy. Email: fdemontis@unica.it, cornelis110553@gmail.com 
Equation (1.1) represents the natural generalization of the matrix NLS equation, i.e.,

$$
i Q_{t}+Q_{x x}-2 \mu Q Q^{\dagger} Q=0_{2 \times 2}, \quad \mu= \pm 1,
$$

where $\mu=-1$ is the focusing case corresponding to case 2 and $\mu=1$ is the defocusing case equivalent to case 1. Equation (1.2) plays an important role in various applicative contexts and, in particular, in the description of Bose-Einstein condensates $[18,19]$. The choice $\mu=1$ (defocusing case) corresponds to repulsive interatomic interactions and antiferromagnetic spinexchange interactions, while the choice $\mu=-1$ (focusing case) takes into account attractive interatomic interactions and ferromagnetic spin-exchange interactions.

The cases 3 and 4 of (1.1) discussed above arise in a natural way in various models of nonlinear optics $[15,16,20]$ and four-component fermionic condensates $[17,26,12,25]$. More specifically, in $[15,16]$ the nonlinear dynamics of the energy transfer process between the fundamental and second harmonic fields in the presence of the phase matched direct current field has been studied in detail; the arising spatio-temporal phenomena can be modeled by cases 3 and 4 of equation (1.1). On the other hand, in [20] cases 3 and 4 of equation (1.1) have been used to model various multicolor optical spatio-temporal solitary waves created by the interaction of light at a central frequency with two sideband waves, both through cross-phase modulation and parametric four-wave mixing of opposite signs. Moreover, cases 3 and 4 of (1.1) appear in the context of fermionic condensates of ultracold atoms and, in particular, such cases allow to investigate four component spin- $\frac{3}{2}$ cold atomic systems under special conditions (see [17, 26, 12, 25]).

Taking into account the important applicative contexts where (1.1) appears, in [22] the Inverse Scattering Transform (IST) for (1.1) has been developed as a tool to solve the associated initial value problem with vanishing boundary conditions, i.e. $Q(x, t) \rightarrow 0_{2 \times 2}$ as $x \rightarrow \pm \infty$ for each fixed $t$, which we assume from now on. While the IST for the cases 1 and 2 is well established $[1,13,2]$, both with zero and nonzero boundary conditions, the IST and soliton solutions corresponding to the reductions 3 and 4 are novel [22], apart from the profound analysis of the one-soliton solutions in cases 2-4 given in [22]. We use the results on the IST obtained in $[22,21]$ to derive a general reflectionless solution formula for (1.1). In fact, in [22] the soliton solutions of (1.1) have been derived by solving the Riemann-Hilbert problem, also in the one-soliton case. Some soliton solutions for equation (1.2) 
have been derived by Ieda et al. [23] by solving the Marchenko equation with nonvanishing boundary conditions. In the present paper we formulate the inverse scattering problem in terms of the Marchenko integral equations, but unlike in [23] we find an explicit reflectionless solution formula by using the so-called matrix triplet method (see, for example, $[4,11,24,7,8]$ for more details on this method). As a consequence of applying the matrix triplet method, we get an explicit reflectionless solution formula comprising not only the soliton solutions already known in the literature but also a new class of explicit soliton solutions such as the two soliton solutions for cases 3 and 4 as well as the double poles soliton solutions for the same cases. It is also important to remark that in this article we verify by algebraic means that our solution formula satisfies (1.1).

Let us briefly discuss the contents of this article. In Section 2 we summarize the well established direct scattering theory of the $2+2$ AKNS system $[1,13,2]$ and discuss the symmetries of Jost solutions and scattering coefficients in all four cases. In Section 3 we derive the Marchenko integral equations and discuss how to compute the potential from the scattering data. In Section 4 we compute the reflectionless solutions by using the matrix triplet method. In this way we generalize the soliton solutions obtained in the focusing case [11] to all four cases, where we take into account both the adjoint symmetry underlying the cases $1-4$ and the assumption that $Q(x, t)$ is a complex symmetric matrix. In Section 5 we substitute the reflectionless solution formula obtained back into the matrix NLS equation (1.1) and verify that it really is a solution. Section 6 is devoted to some illustrative examples, where we recover the well-known one-soliton solutions [22] by other means. The derivation of the Volterra integral equations for the Fourier transforms of the Jost solutions is given in Appendix A.

\section{Direct scattering}

In this section we describe the direct scattering theory of the $2 \times 2$ AKNS system, where the potential $\boldsymbol{Q}(x, t)$ satisfies the symmetry relation underlying the case 1-4 under consideration. At each occasion we point out when the second symmetry of having $Q(x, t)$ complex symmetric is being used. 


\section{$2.1 \quad$ Jost solutions}

It is well-known that the matrix NLS equation is equivalent to the compatibility condition of a Lax pair for the potential matrix $Q(x, t)$, where the first equation in the Lax pair is the so-called matrix Zakharov-Shabat (ZS) or Ablowitz-Kaup-Newell-Segur (AKNS) system. Specifically, Eq. (1.1) admits the Lax pair

$$
\varphi_{x}=\mathcal{U} \varphi, \quad \varphi_{t}=\mathcal{V} \varphi
$$

where

$\mathcal{U}(x, t, k)=-i k \boldsymbol{\sigma}_{3}+\boldsymbol{Q}(x, t), \quad \mathcal{V}(x, t, k)=-2 i k^{2} \boldsymbol{\sigma}_{3}+2 k \boldsymbol{Q}+i \boldsymbol{\sigma}_{3} \boldsymbol{Q}_{x}-i \boldsymbol{\sigma}_{3} \boldsymbol{Q}^{2}$.

Here $\boldsymbol{Q}(x, t)=\left(\begin{array}{cc}0_{2 \times 2} & Q(x, t) \\ R(x, t) & 0_{2 \times 2}\end{array}\right), \boldsymbol{\sigma}_{3}=I_{2} \oplus\left(-I_{2}\right)$, and $R=\Sigma Q^{\dagger} \Omega$.

As in [22], for $(x, t, k) \in \mathbb{R}^{3}$ we define the Jost eigenfunctions as follows:

$$
\begin{aligned}
& \Phi(x, t, k)=(\phi(x, t, k) \quad \bar{\phi}(x, t, k))=e^{-i \theta(x, t, k) \sigma_{3}}\left[I_{4}+o(1)\right], \quad x \rightarrow-\infty, \\
& \Psi(x, t, k)=(\bar{\psi}(x, t, k) \quad \psi(x, t, k))=e^{-i \theta(x, t, k) \boldsymbol{\sigma}_{3}}\left[I_{4}+o(1)\right], \quad x \rightarrow+\infty,
\end{aligned}
$$

where

$$
\theta(x, t, k)=k(x+2 k t)=k x+2 k^{2} t,
$$

and $\phi(x, t, k)$ and $\bar{\phi}(x, t, k)$ (respectively, $\bar{\psi}(x, t, k)$ and $\psi(x, t, k))$ are $4 \times 2$ matrices, called Jost solutions, comprising the first two and last two columns of the $4 \times 4$ matrix solutions $\Phi(x, t, k)$ (respectively, $\Psi(x, t, k))$. Taking out the asymptotic parts we obtain the modified eigenfunctions

$$
\begin{aligned}
& (M(x, t, k) \quad \bar{M}(x, t, k))=\Phi(x, t, k) e^{i \theta(x, t, k) \boldsymbol{\sigma}_{3}} \sim I_{4}, \quad x \rightarrow-\infty, \\
& (\bar{N}(x, t, k) \quad N(x, t, k))=\Psi(x, t, k) e^{i \theta(x, t, k) \sigma_{3}} \sim I_{4}, \quad x \rightarrow+\infty .
\end{aligned}
$$

Assuming that $\boldsymbol{Q}(x, t)$ has its entries in $L^{1}(\mathbb{R}, d x)$ for each $t \in \mathbb{R}$, we can prove the existence of the Jost eigenfunctions as well as their $k$-analyticity properties from the Volterra integral equations

$$
\begin{aligned}
& (M(x, t, k) \quad \bar{M}(x, t, k))=I_{4}+\int_{-\infty}^{x} d y e^{-i k(x-y) \boldsymbol{\sigma}_{3}} \boldsymbol{Q}(y, t) \times \\
& \times(M(y, t, k) \quad \bar{M}(y, t, k)) e^{-i k(y-x) \sigma_{3}}, \\
& (\bar{N}(x, t, k) \quad N(x, t, k))=I_{4}-\int_{x}^{\infty} d y e^{i k(y-x) \boldsymbol{\sigma}_{3}} \boldsymbol{Q}(y, t) \times \\
& \times(\bar{N}(y, t, k) \quad N(y, t, k)) e^{-i k(y-x) \sigma_{3}} .
\end{aligned}
$$


Using substitution of the so-called triangular representations

$$
\begin{aligned}
& (M(x, t, k) \bar{M}(x, t, k))=I_{4}+\int_{-\infty}^{x} d y(L(x, y, t) \quad \bar{L}(x, y, t)) e^{i k(x-y) \sigma_{3}}, \\
& (\bar{N}(x, t, k) \quad N(x, t, k))=I_{4}+\int_{x}^{\infty} d y(\bar{K}(x, y, t) \quad K(x, y, t)) e^{-i k(y-x) \boldsymbol{\sigma}_{3}}
\end{aligned}
$$

into the Volterra integral equations (2.5) and stripping off the Fourier transforms, we get coupled Volterra integral equations for the kernel functions $L(x, y, t)$ and $\bar{L}(x, y, t)$ and for the kernel functions $\bar{K}(x, y, t)$ and $K(x, y, t)$ for each $(x, t) \in \mathbb{R}^{2}$ which can easily be proven to be uniquely solvable in an $L^{1}$-setting [cf. $[2,24,6]$; also Appendix A]. For each $t \in \mathbb{R}$ we thus obtain for the kernel functions

$$
\begin{aligned}
& \sup _{x \in \mathbb{R}} \int_{-\infty}^{x} d y(\|L(x, y, t)\|+\|\bar{L}(x, y, t)\|)<+\infty, \\
& \sup _{x \in \mathbb{R}} \int_{x}^{\infty} d y(\|\bar{K}(x, y, t)\|+\|K(x, y, t)\|)<+\infty .
\end{aligned}
$$

As an ancillary result [see (A.1) and (A.2)], we obtain the inversion formulas

$$
\begin{aligned}
Q(x, t) & =-2 K^{\mathrm{up}}(x, x, t), & R(x, t)=-2 \bar{K}^{\mathrm{dn}}(x, x, t), \\
Q(x, t) & =2 \bar{L}^{\mathrm{up}}(x, x, t), & R(x, t)=2 L^{\mathrm{dn}}(x, x, t), \\
\int_{x}^{\infty} d z(Q R)(z, t) & =2 \bar{K}^{\mathrm{up}}(x, x, t), & \int_{x}^{\infty} d z(R Q)(z, t)=2 K^{\mathrm{dn}}(x, x, t), \\
\int_{-\infty}^{x} d z(Q R)(z, t) & =2 L^{\mathrm{up}}(x, x, t), & \int_{-\infty}^{x} d z(R Q)(z, t)=2 \bar{L}^{\mathrm{dn}}(x, x, t) .
\end{aligned}
$$

If the entries of $Q(x, t)$ and $Q_{x}(x, t)$ belong to $L^{1}(\mathbb{R}, d x)$ for each $t \in \mathbb{R}$, we can derive (2.8) alternatively by deriving the analog of [22, Eqs. (60)].

For later use we present, for $(x, t, k) \in \mathbb{R}^{3}$, the triangular representations

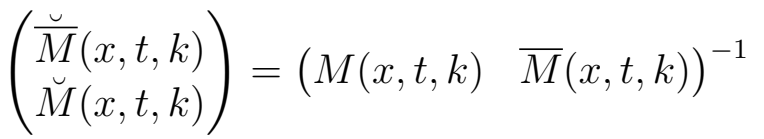

$$
\begin{aligned}
& =I_{4}+\int_{-\infty}^{x} d y e^{-i k(x-y) \boldsymbol{\sigma}_{3}}\left(\begin{array}{l}
\breve{\breve{L}}(x, y, t) \\
\breve{L}(x, y, t)
\end{array}\right),
\end{aligned}
$$




$$
\begin{aligned}
& \left(\begin{array}{l}
\breve{N}(x, t, k) \\
\stackrel{\breve{N}}{\breve{N}}(x, t, k)
\end{array}\right)=(\bar{N}(x, t, k) \quad N(x, t, k))^{-1}
\end{aligned}
$$

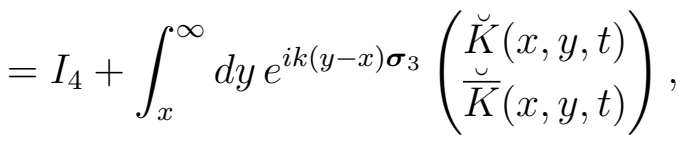

where for each $t \in \mathbb{R}$

$$
\begin{aligned}
& \sup _{x \in \mathbb{R}} \int_{-\infty}^{x} d y(\|\breve{L}(x, y, t)\|+\|\breve{L}(x, y, t)\|)<+\infty, \\
& \sup _{x \in \mathbb{R}} \int_{x}^{\infty} d y(\|\breve{K}(x, y, t)\|+\|\breve{K}(x, y, t)\|)<+\infty .
\end{aligned}
$$

Equations (2.9) can be derived from Volterra integral equations for the inverted Jost eigenfunctions by using the methods explained in Appendix A.

It is easily verified [22] that the $4 \times 4$ potential $\boldsymbol{Q}$ satisfies the symmetry relations

$$
\boldsymbol{Q}^{\dagger}=-\boldsymbol{\Xi}^{-1} \boldsymbol{Q} \boldsymbol{\Xi}, \quad \boldsymbol{\Xi}=\left(\begin{array}{cc}
\Omega^{-1} & 0_{2 \times 2} \\
0_{2 \times 2} & -\Sigma
\end{array}\right)
$$

Equation (2.11) implies the symmetry relations for the Jost eigenfunctions

$$
\begin{aligned}
& \Phi^{-1}(x, t, k)=\boldsymbol{\Xi} \Phi^{\dagger}\left(x, t, k^{*}\right) \boldsymbol{\Xi}^{-1}, \\
& \Psi^{-1}(x, t, k)=\boldsymbol{\Xi} \Psi^{\dagger}\left(x, t, k^{*}\right) \boldsymbol{\Xi}^{-1},
\end{aligned}
$$

where we note that $\theta\left(x, t, k^{*}\right)^{*}=\theta(x, t, k)$.

Also assuming $Q(x, t)$ to be a complex symmetric matrix and putting

$$
\boldsymbol{F}=\left\{\begin{array}{cl}
\left(\begin{array}{cc}
0_{2 \times 2} & -I_{2} \\
I_{2} & 0_{2 \times 2} \\
0_{2 \times 2} & I_{2} \\
-I_{2} & 0_{2 \times 2}
\end{array}\right), \quad \text { cases } 1 \text { and } 3, & \text { cases } 2 \text { and } 4
\end{array}\right.
$$

we obtain the symmetry relations [22]

$$
\begin{aligned}
\Phi^{T}(x, t, k) \boldsymbol{F} \Phi(x, t, k) & =\boldsymbol{F}, \\
\Psi^{T}(x, t, k) \boldsymbol{F} \Psi(x, t, k) & =\boldsymbol{F},
\end{aligned}
$$

where $(x, t, k) \in \mathbb{R}^{3}$. 


\subsection{Scattering coefficients}

Since $\mathcal{U}$ has zero trace and hence $\operatorname{det} \Phi(x, t, k)=\operatorname{det} \Psi(x, t, k)=1$ for $(x, t, k) \in \mathbb{R}^{3}$, there exists a $4 \times 4$ scattering matrix $S(k)$ independent of $(x, t)$ such that

$$
\Phi(x, t, k)=\Psi(x, t, k) S(k) .
$$

Thus we can write in terms of $2 \times 2$ blocks

$$
S(k)=\left(\begin{array}{cc}
a(k) & \bar{b}(k) \\
b(k) & \bar{a}(k)
\end{array}\right), \quad S(k)^{-1}=\left(\begin{array}{cc}
\bar{c}(k) & d(k) \\
\bar{d}(k) & c(k)
\end{array}\right)
$$

where $\operatorname{det} S(k)=1$ for every $k \in \mathbb{R}$. The four diagonal blocks in $S(k)$ and $S(k)^{-1}$ tend to $I_{2}$ and the four off-diagonal blocks vanish as $k \rightarrow \pm \infty$. Using (2.2), (2.6), (2.9), and (2.14) we obtain for the four diagonal blocks $a=\breve{N} M$, $\bar{a}=\breve{N} \bar{M}, \bar{c}=\breve{M} \bar{N}$, and $c=\breve{M} N$. Hence, by virtue of (2.6) and (2.9), there exist matrix functions $\hat{a}, \hat{\bar{a}}, \hat{\bar{c}}$, and $\hat{c}$ such that

$$
\begin{array}{ll}
a(k)=I_{2}+\int_{0}^{\infty} d y e^{i k y} \hat{a}(y), & \bar{a}(k)=I_{2}+\int_{0}^{\infty} d y e^{-i k y} \hat{\bar{a}}(y), \\
\bar{c}(k)=I_{2}+\int_{0}^{\infty} d y e^{-i k y} \hat{\bar{c}}(y), & c(k)=I_{2}+\int_{0}^{\infty} d y e^{i k y} \hat{c}(y),
\end{array}
$$

where

$$
\int_{0}^{\infty} d y(\|\hat{a}(y)\|+\|\hat{\bar{a}}(y)\|+\|\hat{\bar{c}}(y)\|+\|\hat{c}(y)\|)<+\infty .
$$

In the same way, using $e^{2 i \theta} b=\breve{\sim} M, e^{-2 i \theta} \bar{b}=\breve{N} \bar{M}, e^{2 i \theta} \bar{d}=\breve{M} \bar{N}$, and $e^{-2 i \theta} d=\breve{\breve{M}} N$, we prove the existence of matrix functions $\hat{b}, \hat{\bar{b}}, \hat{\bar{d}}$, and $\hat{d}$ such that

$$
\begin{aligned}
& e^{4 i k^{2} t} b(k)=\int_{-\infty}^{\infty} d y e^{-i k y} \hat{b}(y, t), \quad e^{-4 i k^{2} t} \bar{b}(k)=\int_{-\infty}^{\infty} d y e^{i k y} \hat{\bar{b}}(y, t), \\
& e^{4 i k^{2} t} \bar{d}(k)=\int_{-\infty}^{\infty} d y e^{-i k y} \hat{\bar{d}}(y, t), \quad e^{-4 i k^{2} t} d(k)=\int_{-\infty}^{\infty} d y e^{i k y} \hat{d}(y, t)
\end{aligned}
$$

where for each $t \in \mathbb{R}$

$$
\int_{-\infty}^{\infty} d y(\|\hat{b}(y, t)\|+\|\hat{\bar{b}}(y, t)\|+\|\hat{\bar{d}}(y, t)\|+\|\hat{d}(y, t)\|)<+\infty .
$$


Using (2.12) we obtain the symmetry relations

$$
S(k)^{-1}=\Xi S(k)^{\dagger} \Xi^{-1}=\boldsymbol{F}^{-1} S(k)^{T} \boldsymbol{F}, \quad k \in \mathbb{R},
$$

where the transposition symmetry requires $Q$ to be a complex symmetric matrix. These symmetry relations can obviously be transformed into symmetry relations for their separate $2 \times 2$ entries.

Using $S S^{-1}=S^{-1} S=I_{4}$ in (2.15), it is easily verified that $\operatorname{det} a(k)=$ $\operatorname{det} c(k)$ and $\operatorname{det} \bar{a}(k)=\operatorname{det} \bar{c}(k)$. In case 1 (defocusing case) these determinants are positive numbers for each $k \in \mathbb{R}$. In this article we assume that in cases 2-4 these determinants are nonzero for any $k \in \mathbb{R}$ as well. In other words, we assume the absence of spectral singularities. We then define the (matrix) reflection coefficients as follows:

$$
\begin{array}{ll}
\rho(k)=b(k) a(k)^{-1}, & \bar{\rho}(k)=\bar{b}(k) \bar{a}(k)^{-1}, \\
r(k)=d(k) c(k)^{-1}, & \bar{r}(k)=\bar{d}(k) \bar{c}(k)^{-1} .
\end{array}
$$

Under the absence of spectral singularities, there exist matrix functions $\hat{\rho}, \hat{\bar{\rho}}$, $\hat{\bar{r}}$, and $\hat{r}$ such that

$$
\begin{array}{ll}
e^{4 i k^{2} t} \rho(k)=\int_{-\infty}^{\infty} d y e^{-i k y} \hat{\rho}(y, t), & e^{-4 i k^{2} t} \bar{\rho}(k)=\int_{-\infty}^{\infty} d y e^{i k y} \hat{\bar{\rho}}(y, t), \\
e^{4 i k^{2} t} \bar{r}(k)=\int_{-\infty}^{\infty} d y e^{-i k y} \hat{\bar{r}}(y, t), & e^{-4 i k^{2} t} r(k)=\int_{-\infty}^{\infty} d y e^{i k y} \hat{r}(y, t),
\end{array}
$$

where for each $t \in \mathbb{R}$

$$
\int_{-\infty}^{\infty} d y(\|\hat{\rho}(y, t)\|+\|\hat{\bar{\rho}}(y, t)\|+\|\hat{\bar{r}}(y, t)\|+\|\hat{r}(y, t)\|)<+\infty .
$$

Using (2.16), (2.17), and $S S^{-1}=S^{-1} S=I_{4}$, we obtain

$$
\begin{aligned}
& \rho^{\dagger}=\Omega \bar{\rho} \Sigma, \quad \bar{\rho}^{\dagger}=\Sigma^{-1} \rho \Omega^{-1}, \quad \bar{r}^{\dagger}=\Omega r \Sigma, \quad r^{\dagger}=\Sigma^{-1} \bar{r} \Omega^{-1}, \\
& \rho^{T}=\rho, \quad \bar{\rho}^{T}=\bar{\rho}, \quad \bar{r}^{T}=\bar{r}, \quad r^{T}=r,
\end{aligned}
$$

where we have not written the $k$-dependence and $(2.19 \mathrm{~b})$ requires $Q(x, t)$ to be complex symmetric. Equations (2.18) and (2.19a) imply that for a.e. $(y, t) \in \mathbb{R}^{2}$

$$
\begin{array}{llrl}
\hat{\rho}(y ; t)^{\dagger} & =\Omega \hat{\bar{\rho}}(y ; t) \Sigma, & & \hat{\bar{\rho}}(y ; t)^{\dagger}=\Sigma^{-1} \hat{\rho}(y ; t) \Omega^{-1}, \\
\hat{\bar{r}}(y ; t)^{\dagger}=\Omega \hat{r}(y ; t) \Sigma, & & \hat{r}(y ; t)^{\dagger}=\Sigma^{-1} \hat{\bar{r}}(y ; t) \Omega^{-1} .
\end{array}
$$

Using (2.18) and (2.19b), it is clear that $\hat{\rho}, \hat{\bar{\rho}}, \hat{\bar{r}}$, and $\hat{r}$ are complex symmetric matrices whenever $Q(x, t)$ is a complex symmetric matrix. 


\section{Marchenko equations}

In this section we derive the Marchenko integral equations from RiemannHilbert problems for the Jost solutions. We only give full details if the discrete eigenvalues are algebraically and geometrically simple.

\subsection{Riemann-Hilbert problems}

Assuming the absence of spectral singularities and the definitions (2.17) we write the $4 \times 2$ columns of the identities $\Phi=\Psi S$ and $\Psi=\Phi S^{-1}$ in the form

$$
\begin{aligned}
\bar{\psi}(x, t, k) & =\phi(x, t, k) a(k)^{-1}-\psi(x, t, k) \rho(k), \\
\psi(x, t, k) & =\bar{\phi}(x, t, k) \bar{a}(k)^{-1}-\bar{\psi}(x, t, k) \bar{\rho}(k), \\
\phi(x, t, k) & =\bar{\psi}(x, t, k) \bar{c}(k)^{-1}-\bar{\phi}(x, t, k) \bar{r}(k), \\
\bar{\phi}(x, t, k) & =\psi(x, t, k) c(k)^{-1}-\phi(x, t, k) r(k) .
\end{aligned}
$$

Replacing Jost functions by modified Jost functions and rearranging terms we obtain

$$
\begin{aligned}
& M(x, t, k) a(k)^{-1}-\left(\begin{array}{c}
I_{2} \\
0_{2 \times 2}
\end{array}\right)=\bar{N}(x, t, k)-\left(\begin{array}{c}
I_{2} \\
0_{2 \times 2}
\end{array}\right)+e^{2 i \theta(x, t, k)} N(x, t, k) \rho(k), \\
& \bar{M}(x, t, k) \bar{a}(k)^{-1}-\left(\begin{array}{c}
0_{2 \times 2} \\
I_{2}
\end{array}\right)=N(x, t, k)-\left(\begin{array}{c}
0_{2 \times 2} \\
I_{2}
\end{array}\right)+e^{-2 i \theta(x, t, k) \bar{N}}(x, t, k) \bar{\rho}(k), \\
& \bar{N}(x, t, k) \bar{c}(k)^{-1}-\left(\begin{array}{c}
I_{2} \\
0_{2 \times 2}
\end{array}\right)=M(x, t, k)-\left(\begin{array}{c}
I_{2} \\
0_{2 \times 2}
\end{array}\right)+e^{2 i \theta(x, t, k)} \bar{M}(x, t, k) \bar{r}(k), \\
& N(x, t, k) c(k)^{-1}-\left(\begin{array}{c}
0_{2 \times 2} \\
I_{2}
\end{array}\right)=\bar{M}(x, t, k)-\left(\begin{array}{c}
0_{2 \times 2} \\
I_{2}
\end{array}\right)+e^{-2 i \theta(x, t, k)} M(x, t, k) r(k) .
\end{aligned}
$$

Thus either side of each of (3.2) is the Fourier transform of a $4 \times 2$ matrix function with entries in $L^{1}(\mathbb{R})$.

Letting $\mathcal{W}, \mathcal{W}_{+}$, and $\mathcal{W}_{-}$stand for the Fourier transforms of the $L^{1}$ 
functions supported on $\mathbb{R}, \mathbb{R}^{+}$, and $\mathbb{R}^{-}$, we get the commutative diagram

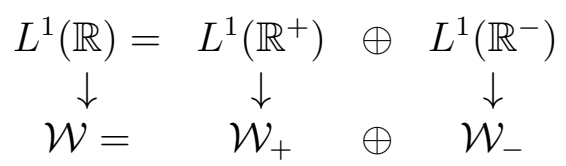

where the downarrows represent the Fourier transform $\mathcal{F}$ defined by

$$
(\mathcal{F} f)(k)=\int_{-\infty}^{\infty} d y e^{i k y} f(y)
$$

and the norms on $\mathcal{W}, \mathcal{W}_{+}$, and $\mathcal{W}_{-}$are defined in such a way that the downarrows become isometries. Then the complementary projections $\Pi_{ \pm}$of $\mathcal{W}$ onto $\mathcal{W}_{ \pm}$along $\mathcal{W}_{\mp}$ are given by the Plemelj formulas

$$
\left(\Pi_{ \pm} f\right)(k)=\frac{ \pm 1}{2 \pi i} \int_{-\infty}^{\infty} d \xi \frac{f(\xi)}{\xi-\left(k \pm i 0^{+}\right)}, \quad f \in \mathcal{W} .
$$

Applying the projection $\Pi_{-}$to $(3.2 \mathrm{a})$ and $(3.2 \mathrm{~d})$ and the projection $\Pi_{+}$to $(3.2 \mathrm{~b})$ and $(3.2 \mathrm{c})$, we arrive at $4 \times 2$ matrix singular integral equations (usually called Riemann-Hilbert problems in the integrable systems literature) which couple all four functions $N, \bar{N}, \bar{M}$, and $M$. To arrive at two equations coupling $N$ and $\bar{N}$ and two equations coupling $\bar{M}$ and $M$, we introduce the so-called norming constants.

If all of the poles $i k_{j}$ in the upper half-plane $\mathbb{C}^{+}$are simple, letting $\tau_{j}$ and $\breve{\tau}_{j}$ be the residues of $a(k)^{-1}$ and $c(k)^{-1}$ at $k=i k_{j}$ and $\bar{\tau}_{j}$ and $\breve{\bar{\tau}}_{j}$ the residues of $\bar{a}(k)^{-1}$ and $\bar{c}(k)^{-1}$ at $k=-i k_{j}^{*}$, we define the norming constants $C_{j}, \bar{C}_{j}, D_{j}$, and $\bar{D}_{j}$ by rewriting the residues of the left-hand sides of (3.2) as follows:

$$
\begin{aligned}
M\left(x, t, i k_{j}\right) \tau_{j} & =e^{2 i \theta\left(x, t, i k_{j}\right)} N\left(x, t, i k_{j}\right) C_{j}, \\
\bar{M}\left(x, t,-i k_{j}^{*}\right) \bar{\tau}_{j} & =e^{-2 i \theta\left(x, t,-i k_{j}^{*}\right)} \bar{N}\left(x, t,-i k_{j}^{*}\right) \bar{C}_{j}, \\
\bar{N}\left(x, t,-i k_{j}^{*}\right) \breve{\bar{\tau}}_{j} & =e^{2 i \theta\left(x, t,-i k_{j}^{*}\right)} \bar{M}\left(x, t,-i k_{j}^{*}\right) \bar{D}_{j}, \\
N\left(x, t, i k_{j}\right) \breve{\tau}_{j} & =e^{-2 i \theta\left(x, t, i k_{j}\right)} M\left(x, t, i k_{j}\right) D_{j} .
\end{aligned}
$$

As a result, we get

$$
\begin{aligned}
\sum_{j} \frac{e^{2 i \theta\left(x, t, i k_{j}\right)} N\left(x, t, i k_{j}\right) C_{j}}{k-i k_{j}} & =\bar{N}(x, t, k)-\left(\begin{array}{c}
I_{2} \\
0_{2 \times 2}
\end{array}\right) \\
& -\int_{-\infty}^{\infty} \frac{d \xi}{2 \pi i} \frac{e^{2 i \theta(x, t, \xi)} N(x, t, \xi) \rho(\xi)}{\xi-k+i 0^{+}}
\end{aligned}
$$




$$
\begin{aligned}
\sum_{j} \frac{e^{-2 i \theta\left(x, t,-i k_{j}^{*}\right)} \bar{N}\left(x, t,-i k_{j}^{*}\right) \bar{C}_{j}}{k+i k_{j}^{*}} & =N(x, t, k)-\left(\begin{array}{c}
0_{2 \times 2} \\
I_{2}
\end{array}\right) \\
& +\int_{-\infty}^{\infty} \frac{d \xi}{2 \pi i} \frac{e^{-2 i \theta(x, t, \xi)} \bar{N}(x, t, \xi) \bar{\rho}(\xi)}{\xi-k-i 0^{+}} \\
\sum_{j} \frac{e^{2 i \theta\left(x, t,-i k_{j}^{*}\right)} \bar{M}\left(x, t,-i k_{j}^{*}\right) \bar{D}_{j}}{k+i k_{j}^{*}} & =M(x, t, k)-\left(\begin{array}{c}
I_{2} \\
0_{2 \times 2}
\end{array}\right) \\
& +\int_{-\infty}^{\infty} \frac{d \xi}{2 \pi i} \frac{e^{2 i \theta(x, t, \xi)} \bar{M}(x, t, \xi) \bar{r}(\xi)}{\xi-k-i 0^{+}} \\
\sum_{j} \frac{e^{-2 i \theta\left(x, t, i k_{j}\right)} M\left(x, t, i k_{j}\right) D_{j}}{k-i k_{j}} & =\bar{M}(x, t, k)-\left(\begin{array}{c}
0_{2 \times 2} \\
I_{2}
\end{array}\right) \\
& -\int_{-\infty}^{\infty} \frac{d \xi}{2 \pi i} \frac{e^{-2 i \theta(x, t, \xi)} M(x, t, \xi) r(\xi)}{\xi-k+i 0^{+}}
\end{aligned}
$$

Substituting $k=i k_{j}$ in (3.5b) and (3.5c) and $k=-i k_{j}^{*}$ in (3.5a) and (3.5d), we obtain linear systems for $N\left(x, t, i k_{j}\right)$ and $M\left(x, t, i k_{j}\right)$ and for $\bar{N}\left(x, t,-i k_{j}^{*}\right)$ and $\bar{M}\left(x, t,-i k_{j}^{*}\right)$, respectively, which enable us to compute the modified Jost solutions. These linear constraints are equivalent to the usual analyticity requirements on the modified Jost solutions.

If there is a pole of order $m_{j}$ at $i k_{j}$, we define the corresponding norming constants $C_{j, s}$ and $D_{j, s}\left(s=0,1, \ldots, m_{j}-1\right)$ in such a way that the expressions

$$
\begin{gathered}
M(x, t, k) a(k)^{-1}-e^{2 i \theta(x, t, k)} N(x, t, k) \sum_{s=0}^{m_{j}-1} \frac{C_{j, s}}{\left(k-i k_{j}\right)^{s+1}}, \\
N(x, t, k) c(k)^{-1}-e^{-2 i \theta(x, t, k)} M(x, t, k) \sum_{s=0}^{m_{j}-1} \frac{D_{j, s}}{\left(k-i k_{j}\right)^{s+1}},
\end{gathered}
$$

are analytic in a neighbourhood of $k=i k_{j}$. In a similar way we define the corresponding norming constants $\bar{C}_{j, s}$ and $\bar{D}_{j, s}\left(s=0,1, \ldots, m_{j}-1\right)$ in such 
a way that the expressions

$$
\begin{gathered}
\bar{M}(x, t, k) a(k)^{-1}-e^{2 i \theta(x, t, k)} \bar{N}(x, t, k) \sum_{s=0}^{m_{j}-1} \frac{\bar{C}_{j, s}}{\left(k+i k_{j}^{*}\right)^{s+1}}, \\
\bar{N}(x, t, k) c(k)^{-1}-e^{-2 i \theta(x, t, k)} \bar{M}(x, t, k) \sum_{s=0}^{m_{j}-1} \frac{\bar{D}_{j, s}}{\left(k+i k_{j}^{*}\right)^{s+1}},
\end{gathered}
$$

are analytic in a neighborhood of $k=-i k_{j}^{*}$. The resulting generalizations of (3.5) can then be used to derive linear constraints involving the values and $k$-derivatives of the modified Jost solutions up to the order $m_{j}-1$ which are equivalent to the usual analyticity properties of the modified Jost solutions ([22, Appendix B] for double poles).

\subsection{Derivation of Marchenko equations}

The Marchenko integral equations are equivalent to the Riemann-Hilbert problems (3.5) (and their multipole analogs). Replacing the modified Jost solutions $M, \bar{M}, \bar{N}$, and $N$ by their corresponding kernel functions $L, \bar{L}, \bar{K}$, and $K$ by using the triangular representations and stripping off the Fourier transform, the Marchenko integral equations appear as a result. Instead of considering the Riemann-Hilbert problems in $\mathcal{W}_{-}^{4 \times 2}, \mathcal{W}_{+}^{4 \times 2}, \mathcal{W}_{+}^{4 \times 2}$, and $\mathcal{W}_{-}^{4 \times 2}$, we study the Marchenko equations in $L^{1}(-\infty, x)^{4 \times 2}, L^{1}(x,+\infty)^{4 \times 2}$, $L^{1}(x,+\infty)^{4 \times 2}$, and $L^{1}(-\infty, x)^{4 \times 2}$, respectively.

Equations (2.6) imply

$$
\begin{aligned}
& \bar{K}(x, y, t)=\frac{1}{2 \pi} \int_{-\infty}^{\infty} d k e^{i k(y-x)}\left[\bar{N}(x, t, k)-\left(\begin{array}{c}
I_{2} \\
0_{2 \times 2}
\end{array}\right)\right], \\
& K(x, y, t)=\frac{1}{2 \pi} \int_{-\infty}^{\infty} d k e^{i k(x-y)}\left[N(x, t, k)-\left(\begin{array}{c}
0_{2 \times 2} \\
I_{2}
\end{array}\right)\right], \\
& L(x, y, t)=\frac{1}{2 \pi} \int_{-\infty}^{\infty} d k e^{i k(y-x)}\left[M(x, t, k)-\left(\begin{array}{c}
I_{2} \\
0_{2 \times 2}
\end{array}\right)\right], \\
& \bar{L}(x, y, t)=\frac{1}{2 \pi} \int_{-\infty}^{\infty} d k e^{i k(x-y)}\left[\bar{M}(x, t, k)-\left(\begin{array}{c}
0_{2 \times 2} \\
I_{2}
\end{array}\right)\right] .
\end{aligned}
$$

Applying $\frac{1}{2 \pi} \int_{-\infty}^{\infty} d k e^{i k(y-x)}$ to (3.5a) and (3.5c) and $\frac{1}{2 \pi} \int_{-\infty}^{\infty} d k e^{i k(x-y)}$ to $(3.5 \mathrm{~b})$ and $(3.5 \mathrm{~d})$, we obtain the Marchenko integral equations (see, for ex- 
ample, [7] for more details on the following equations)

$$
\begin{gathered}
\bar{K}(x, y, t)+\left(\begin{array}{c}
0_{2 \times 2} \\
F_{r}(x+y, t)
\end{array}\right)+\int_{x}^{\infty} d z K(x, z, t) F_{r}(z+y, t)=0_{4 \times 2}, \\
K(x, y, t)+\left(\begin{array}{c}
\bar{F}_{r}(x+y, t) \\
0_{2 \times 2}
\end{array}\right)+\int_{x}^{\infty} d z \bar{K}(x, z, t) \bar{F}_{r}(z+y, t)=0_{4 \times 2}, \\
L(x, y, t)+\left(\begin{array}{c}
0_{2 \times 2} \\
\bar{F}_{l}(x+y, t)
\end{array}\right)+\int_{-\infty}^{x} d z \bar{L}(x, z, t) \bar{F}_{l}(z+y, t)=0_{4 \times 2}, \\
\bar{L}(x, y, t)+\left(\begin{array}{c}
F_{l}(x+y, t) \\
0_{2 \times 2}
\end{array}\right)+\int_{-\infty}^{x} d z L(x, z, t) F_{l}(z+y, t)=0_{4 \times 2},
\end{gathered}
$$

where the Marchenko kernels are given by

$$
\begin{aligned}
& F_{r}(w, t)=\hat{\rho}(w, t)-i \sum_{j} e^{-k_{j} w} e^{-4 i k_{j}^{2} t} C_{j}, \\
& \bar{F}_{r}(w, t)=\hat{\bar{\rho}}(w, t)+i \sum_{j} e^{-k_{j}^{*} w} e^{4 i k_{j}^{* 2} t} \bar{C}_{j}, \\
& \bar{F}_{l}(w, t)=\hat{\bar{r}}(w, t)+i \sum_{j} e^{k_{j}^{*} w} e^{-4 i k_{j}^{* 2} t} \bar{D}_{j}, \\
& F_{l}(w, t)=\hat{r}(w, t)-i \sum_{j} e^{k_{j} w} e^{4 i k_{j}^{2} t} D_{j} .
\end{aligned}
$$

The Marchenko integral kernels $F_{r}, \bar{F}_{r}, \bar{F}_{l}$, and $F_{l}$ are complex symmetric matrices if $Q$ is a complex symmetric matrix. Moreover, in this case

$$
\begin{array}{ll}
F_{r}(w, t)^{\dagger}=\Omega \bar{F}_{r}(w, t) \Sigma, & \bar{F}_{r}(w, t)^{\dagger}=\Sigma^{-1} F_{r}(w, t) \Omega^{-1}, \\
\bar{F}_{l}(w, t)^{\dagger}=\Omega F_{l}(w, t) \Sigma, & F_{l}(w, t)^{\dagger}=\Sigma^{-1} \bar{F}_{l}(w, t) \Omega^{-1},
\end{array}
$$

since the Marchenko integral kernels satisfy the same conjugation and transposition properties as the corresponding Fourier transposed reflection coefficients $[10,8]$. Thus, for complex symmetric potentials $Q(x, t)$ the norming constants $C_{j}, \bar{C}_{j}, \bar{D}_{j}$, and $D_{j}$ are complex symmetric matrices satisfying

$$
C_{j}^{\dagger}=\Omega \bar{C}_{j} \Sigma, \quad D_{j}^{\dagger}=\Sigma^{-1} \bar{D}_{j} \Omega^{-1} .
$$

Similar properties hold for the generalized norming constants in the case of multiple poles.

The principle of generalizing the symmetries of the reflection coefficients to identical symmetries for the corresponding Marchenko integral kernels tells us that the four Marchenko integral kernels are complex symmetric $2 \times 2$ matrices. A rigorous proof of the above principle can be found in $[10,8]$. 


\section{Matrix triplet method}

In this section we employ the matrix triplet method to express the reflectionless NLS solutions in a triplet of three matrices. The impact on these triplets of having a complex symmetric potential $Q(x, t)$ is discussed at the end of this section.

\subsection{Marchenko kernels and kernel functions}

Let us assume that the reflection coefficients $\rho, \bar{\rho}, \bar{r}$, and $r$ vanish identically. Then the Marchenko integral kernels have separated variables. We can then introduce the four matrix triplets $\left(A_{r}, B_{r}, C_{r}\right),\left(\bar{A}_{r}, \bar{B}_{r}, \bar{C}_{r}\right),\left(\bar{A}_{l}, \bar{B}_{l}, \bar{C}_{l}\right)$, and $\left(A_{l}, B_{l}, C_{l}\right)$ such that

$$
\begin{aligned}
F_{r}(w, t) & =C_{r} e^{-w A_{r}} e^{-4 i t A_{r}^{2}} B_{r}, \\
\bar{F}_{r}(w, t) & =\bar{C}_{r} e^{-w \bar{A}_{r}} e^{4 i t \bar{A}_{r}^{2}} \bar{B}_{r}, \\
\bar{F}_{l}(w, t) & =\bar{C}_{l} e^{w \bar{A}_{l}} e^{-4 i t \bar{A}_{l}^{2}} \bar{B}_{l}, \\
F_{l}(w, t) & =C_{l} e^{w A_{l}} e^{4 i t A_{l}^{2}} B_{l} .
\end{aligned}
$$

The four Marchenko kernels are complex symmetric $2 \times 2$ matrices and each triplet is such that the dimension of the matrices $A_{r, l}\left(\bar{A}_{r, l}\right), C_{r, l}\left(\bar{C}_{r, l}\right)$ and $B_{r, l}\left(\bar{B}_{r, l}\right)$ are, respectively, $p \times p, 2 \times p$ and $p \times 2$. If the triplet $\left(A_{r}, B_{r}, C_{r}\right)$ is minimal in the sense of representing $F_{r}(w, t)$ using matrices of minimal sizes, then there exists a complex symmetric invertible matrix $\mathcal{S}_{r}$ such that $\mathcal{S}_{r} A_{r}=A_{r}^{T} \mathcal{S}_{r}, \mathcal{S}_{r} B_{r}=C_{r}^{T}$, and $C_{r} \mathcal{S}_{r}^{-1}=B_{r}^{T}$ (cf. [5]). Analogous properties hold for the other three matrix triplets when minimal.

Equations (3.7) imply

$$
\begin{aligned}
\bar{K}(x, y, t) & =-W_{r}(x, t) e^{-4 i t A_{r}^{2}} e^{-y A_{r}} B_{r}, \\
K(x, y, t) & =-\bar{W}_{r}(x, t) e^{4 i t \bar{A}_{r}^{2}} e^{-y \bar{A}_{r}} \bar{B}_{r} \\
L(x, y, t) & =-\bar{W}_{l}(x, t) e^{-4 i t \bar{A}_{l}^{2}} e^{y \bar{A}_{l}} \bar{B}_{l} \\
\bar{L}(x, y, t) & =-W_{l}(x, t) e^{4 i t A_{l}^{2}} e^{y A_{l}} B_{l}
\end{aligned}
$$


where

$$
\begin{aligned}
W_{r}(x, t) & =\left(\begin{array}{c}
0_{2 \times p} \\
C_{r} e^{-x A_{r}}
\end{array}\right)+\int_{x}^{\infty} d z K(x, z, t) C_{r} e^{-z A_{r}}, \\
W_{r}(x, t) & =\left(\begin{array}{c}
\bar{C}_{r} e^{-x \bar{A}_{r}} \\
0_{2 \times p}
\end{array}\right)+\int_{x}^{\infty} d z \bar{K}(x, z, t) \bar{C}_{r} e^{-z \bar{A}_{r}}, \\
\bar{W}_{l}(x, t) & =\left(\begin{array}{c}
0_{2 \times p} \\
\bar{C}_{l} e^{x \bar{A}_{l}}
\end{array}\right)+\int_{-\infty}^{x} d z \bar{L}(x, z, t) \bar{C}_{l} e^{z \bar{A}_{l}}, \\
W_{l}(x, t) & =\left(\begin{array}{c}
C_{l} e^{x A_{l}} \\
0_{2 \times 2}
\end{array}\right)+\int_{-\infty}^{x} d z L(x, z, t) C_{l} e^{z A_{l}} .
\end{aligned}
$$

Introducing the unique solutions

$$
\begin{aligned}
Q_{r} & =\int_{0}^{\infty} d z e^{-z \bar{A}_{r}} \bar{B}_{r} C_{r} e^{-z A_{r}}, & N_{r} & =\int_{0}^{\infty} d z e^{-z A_{r}} B_{r} \bar{C}_{r} e^{-z \bar{A}_{r}}, \\
Q_{l} & =\int_{-\infty}^{0} d z e^{z \bar{A}_{l}} \bar{B}_{l} C_{l} e^{z A_{l}}, & N_{l} & =\int_{-\infty}^{0} d z e^{z A_{l}} B_{l} \bar{C}_{l} e^{z \bar{A}_{l}},
\end{aligned}
$$

of the Sylvester equations

$$
\begin{aligned}
\bar{A}_{r} Q_{r}+Q_{r} A_{r} & =\bar{B}_{r} C_{r}, \quad A_{r} N_{r}+N_{r} \bar{A}_{r}=B_{r} \bar{C}_{r}, \\
\bar{A}_{l} Q_{l}+Q_{l} A_{l} & =\bar{B}_{l} C_{l}, \quad A_{l} N_{l}+N_{l} \bar{A}_{l}=B_{l} \bar{C}_{l},
\end{aligned}
$$

and substituing equations (4.1) and (4.2) in (3.7) we obtain the linear systems

$$
\begin{aligned}
\left(\begin{array}{ll}
\bar{W}_{r}(x, t) & W_{r}(x, t)
\end{array}\right)\left(\begin{array}{cc}
I_{p} & \mathcal{K}_{r}(x, t) \\
\mathcal{J}_{r}(x, t) & I_{p}
\end{array}\right) & =\left(\begin{array}{cc}
\bar{C}_{r} e^{-x \bar{A}_{r}} & 0_{2 \times p} \\
0_{2 \times p} & C_{r} e^{-x A_{r}}
\end{array}\right) \\
\left(\begin{array}{lll}
W_{l}(x, t) & W_{l}(x, t)
\end{array}\right)\left(\begin{array}{cc}
I_{p} & \mathcal{K}_{l}(x, t) \\
\mathcal{J}_{l}(x, t) & I_{p}
\end{array}\right) & =\left(\begin{array}{cc}
C_{l} e^{x A_{l}} & 0_{2 \times p} \\
0_{2 \times p} & \bar{C}_{l} e^{x \bar{A}_{l}}
\end{array}\right)
\end{aligned}
$$

where

$$
\begin{array}{ll}
\mathcal{J}_{r}(x, t)=e^{-4 i t A_{r}^{2}} e^{-x A_{r}} N_{r} e^{-x \bar{A}_{r}}, & \mathcal{K}_{r}(x, t)=e^{4 i t \bar{A}_{r}^{2}} e^{-x \bar{A}_{r}} Q_{r} e^{-x A_{r}}, \\
\mathcal{J}_{l}(x, t)=e^{-4 i t \bar{A}_{l}^{2}} e^{x \bar{A}_{l}} Q_{l} e^{x A_{l}}, & \mathcal{K}_{l}(x, t)=e^{4 i t A_{l}^{2}} e^{x A_{l}} N_{l} e^{x \bar{A}_{l}}
\end{array}
$$


From the systems (4.6a) and (4.6b) we easily get

$$
\begin{aligned}
\bar{W}_{r}(x, t) \bar{\Gamma}_{r}(x, t) & =\left(\begin{array}{c}
\bar{C}_{r} e^{-x \bar{A}_{r}} \\
-C_{r} e^{-2 x A_{r}} e^{-4 i t A_{r}^{2}} N_{r} e^{-x \bar{A}_{r}}
\end{array}\right) \\
W_{r}(x, t) \Gamma_{r}(x, t) & =\left(\begin{array}{c}
-\bar{C}_{r} e^{-2 x \bar{A}_{r}} e^{4 i t A_{r}^{2}} Q_{r} e^{-x A_{r}} \\
C_{r} e^{-x A_{r}}
\end{array}\right) \\
\bar{W}_{l}(x, t) \bar{\Gamma}_{l}(x, t) & =\left(\begin{array}{c}
-C_{l} e^{2 x A_{l}} e^{4 i t A_{l}^{2}} N_{l} e^{x A_{l}} \\
\bar{C}_{l} e^{-2 x \bar{A}_{l}}
\end{array}\right) \\
W_{l}(x, t) \Gamma_{l}(x, t) & =\left(\begin{array}{c}
C_{l} e^{-x A_{l}} \\
-\bar{C}_{l} e^{2 x \bar{A}_{l}} e^{-4 i t \bar{A}_{l}^{2}} Q_{l} e^{x \bar{A}_{l}}
\end{array}\right) .
\end{aligned}
$$

where

$$
\begin{aligned}
& \bar{\Gamma}_{r}(x, t)=I_{p}-e^{4 i t \bar{A}_{r}^{2}} e^{-x \bar{A}_{r}} Q_{r} e^{-4 i t A_{r}^{2}} e^{-2 x A_{r}} N_{r} e^{-x \bar{A}_{r}}, \\
& \Gamma_{r}(x, t)=I_{p}-e^{-4 i t A_{r}^{2}} e^{-x A_{r}} N_{r} e^{4 i t \bar{A}_{r}^{2}} e^{-2 x \bar{A}_{r}} Q_{r} e^{-x A_{r}}, \\
& \Gamma_{l}(x, t)=I_{p}-e^{4 i t A_{l}^{2}} e^{x A_{l}} N_{l} e^{-4 i t \bar{A}_{l}^{2}} e^{2 x \bar{A}_{l}} Q_{l} e^{x A_{l}}, \\
& \bar{\Gamma}_{l}(x, t)=I_{p}-e^{-4 i t \bar{A}_{l}^{2}} e^{x \bar{A}_{l}} Q_{l} e^{4 i t A_{l}^{2}} e^{2 x A_{l}} N_{l} e^{x \bar{A}_{l}},
\end{aligned}
$$

Then, from equations (4.2) we arrive at the following

$$
\begin{aligned}
& \bar{K}^{\mathrm{up}}(x, y, t)=\bar{C}_{r} e^{-2 x \bar{A}_{r}} e^{4 i t \bar{A}_{r}^{2}} Q_{r} e^{-x A_{r}} \Gamma_{r}(x, t)^{-1} e^{-4 i t A_{r}^{2}} e^{-y A_{r}} B_{r}, \\
& \bar{K}^{\mathrm{dn}}(x, y, t)=-C_{r} e^{-x A_{r}} \Gamma_{r}(x, t)^{-1} e^{-4 i t A_{r}^{2}} e^{-y A_{r}} B_{r}, \\
& K^{\mathrm{up}}(x, y, t)=-\bar{C}_{r} e^{-x \bar{A}_{r}} \bar{\Gamma}_{r}(x, t)^{-1} e^{4 i t \bar{A}_{r}^{2}} e^{-y \bar{A}_{r}} \bar{B}_{r}, \\
& K^{\mathrm{dn}}(x, y, t)=C_{r} e^{-2 x A_{r}} e^{-4 i t A_{r}^{2}} N_{r} e^{-x \bar{A}_{r}} \bar{\Gamma}_{r}(x, t)^{-1} e^{4 i t \bar{A}_{r}^{2}} e^{-y \bar{A}_{r}} \bar{B}_{r},
\end{aligned}
$$

provided the matrix inverses exist. Proceeding in a very similar way we get as well

$$
\begin{aligned}
& L^{\mathrm{up}}(x, y, t)=C_{l} e^{2 x A_{l}} e^{4 i t A_{l}^{2}} N_{l} e^{x \bar{A}_{l}} \bar{\Gamma}_{l}(x, t)^{-1} e^{-4 i t \bar{A}_{l}^{2}} e^{y \bar{A}_{l}} \bar{B}_{l}, \\
& L^{\mathrm{dn}}(x, y, t)=-\bar{C}_{l} e^{x \bar{A}_{l}} \bar{\Gamma}_{l}(x, t)^{-1} e^{-4 i t \bar{A}_{l}^{2}} e^{y \bar{A}_{l}} \bar{B}_{l}, \\
& \bar{L}^{\mathrm{up}}(x, y, t)=-C_{l} e^{x A_{l}} \Gamma_{l}(x, t)^{-1} e^{4 i t A_{l}^{2}} e^{y A_{l}} B_{l}, \\
& \bar{L}^{\mathrm{dn}}(x, y, t)=\bar{C}_{l} e^{2 x \bar{A}_{l}} e^{-4 i t \bar{A}_{l}^{2}} Q_{l} e^{x A_{l}} \Gamma_{l}(x, t)^{-1} e^{4 i t A_{l}^{2}} e^{y A_{l}} B_{l},
\end{aligned}
$$


provided the matrix inverses exist. In fact, applying the Sherman-Morrison formula [14] to $\bar{\Gamma}_{r}(x, t)=I_{2}-\mathcal{K}_{r}(x, t) \mathcal{J}_{r}(x, t), \Gamma_{r}(x, t)=I_{2}-\mathcal{J}_{r}(x, t) \mathcal{K}_{r}(x, t)$, $\Gamma_{l}(x, t)=I_{2}-\mathcal{K}_{l}(x, t) \mathcal{J}_{l}(x, t)$, and $\bar{\Gamma}_{l}(x, t)=I_{2}-\mathcal{J}_{l}(x, t) \mathcal{K}_{l}(x, t)$, we obtain

$$
\operatorname{det} \bar{\Gamma}_{r}(x, t)=\operatorname{det} \Gamma_{r}(x, t), \quad \operatorname{det} \bar{\Gamma}_{l}(x, t)=\operatorname{det} \Gamma_{l}(x, t) \text {. }
$$

Matrix triplets where the Sylvester equations (4.5) are uniquely solvable, but $A_{r}, \bar{A}_{r}, \bar{A}_{l}$, or $A_{l}$ may have some eigenvalues with nonpositive real part, can always be transformed into matrix triplets of the above kind without changing the Marchenko kernels [3].

In case 1 (defocusing case) there do not exist any soliton solutions [22]. In the other three cases we replace the overlined quantities by complex conjugate transposes or ordinary transposes using the symmetry relations. In fact [5], if the representations (4.1) are minimal in the sense that $A_{r}, \bar{A}_{r}, \bar{A}_{l}$, and $A_{l}$ have minimal matrix order among all matrix triplets leading to the same Marchenko integral kernels, there exist unique nonsingular matrices $S_{r}$ and $S_{l}$ such that

$$
\begin{array}{lll}
\bar{A}_{r}=S_{r}^{-1} A_{r}^{\dagger} S_{r}, & \bar{B}_{r}=S_{r}^{-1} C_{r}^{\dagger} \Sigma^{-1}, & \bar{C}_{r}=\Omega^{-1} B_{r}^{\dagger} S_{r}, \\
\bar{A}_{l}=S_{l}^{-1} A_{l}^{\dagger} S_{l}, & \bar{B}_{l}=S_{l}^{-1} C_{l}^{\dagger} \Sigma^{-1}, & \bar{C}_{l}=\Omega^{-1} B_{l}^{\dagger} S_{l} .
\end{array}
$$

Then the Lyapunov solutions in (4.4) satisfy

$$
Q_{r}=S_{r}^{-1} Q_{r, \Sigma}, \quad N_{r}=N_{r, \Omega} S_{r}, \quad Q_{l}=S_{l}^{-1} Q_{l, \Sigma}, \quad N_{l}=N_{l, \Omega} S_{l},
$$

where $Q_{r, \Sigma}, N_{r, \Omega}, Q_{l, \Sigma}$, and $N_{l, \Omega}$ are the hermitian matrices given by

$$
\begin{aligned}
Q_{r, \Sigma} & =\int_{0}^{\infty} d z e^{-z A_{r}^{\dagger}} C_{r}^{\dagger} \Sigma^{-1} C_{r} e^{-z A_{r}}, \\
N_{r, \Omega} & =\int_{0}^{\infty} d z e^{-z A_{r}} B_{r} \Omega^{-1} B_{r}^{\dagger} e^{-z A_{r}^{\dagger}}, \\
Q_{l, \Sigma} & =\int_{0}^{\infty} d z e^{-z A_{l}^{\dagger}} C_{l}^{\dagger} \Sigma^{-1} C_{l} e^{-z A_{l}}, \\
N_{l, \Omega} & =\int_{0}^{\infty} d z e^{-z A_{l}} B_{l} \Omega^{-1} B_{l}^{\dagger} e^{-z A_{l}^{\dagger}} .
\end{aligned}
$$

It is readily verified that the expressions in (4.13) are the unique solutions of the respective Lyapunov equations

$$
\begin{aligned}
A_{r}^{\dagger} Q_{r, \Sigma}+Q_{r, \Sigma} A_{r} & =C_{r}^{\dagger} \Sigma^{-1} C_{r}, & A_{r} N_{r, \Omega}+N_{r, \Omega} A_{r}^{\dagger} & =B_{r} \Omega^{-1} B_{r}^{\dagger}, \\
A_{l}^{\dagger} Q_{l, \Sigma}+Q_{l, \Sigma} A_{l} & =C_{l}^{\dagger} \Sigma^{-1} C_{l}, & A_{l} N_{l, \Omega}+N_{l, \Omega} A_{l}^{\dagger} & =B_{l} \Omega^{-1} B_{l}^{\dagger} .
\end{aligned}
$$


Using (4.9), (4.10), (4.11), and (4.12) we obtain

$$
\begin{aligned}
\bar{K}^{\mathrm{up}}(x, y, t) & =\Omega^{-1} B_{r}^{\dagger}\left[e^{-4 i t A_{r}^{\dagger}} e^{2 x A_{r}^{\dagger}}-Q_{r, \Sigma} e^{-4 i t A_{r}^{2}} e^{-2 x A_{r}} N_{r, \Omega}\right]^{-1} \times \\
& \times Q_{r, \Sigma} e^{-4 i t A_{r}^{2}} e^{-(x+y) A_{r}} B_{r} \\
\bar{K}^{\mathrm{dn}}(x, y, t) & =-C_{r}\left[e^{4 i t A_{r}^{2}} e^{2 x A_{r}}-N_{r, \Omega} e^{4 i t A_{r}^{\dagger^{2}}} e^{-2 x A_{r}^{\dagger}} Q_{r, \Sigma}\right]^{-1} \times \\
& \times e^{-(y-x) A_{r}} B_{r} \\
K^{\mathrm{up}}(x, y, t) & =-\Omega^{-1} B_{r}^{\dagger}\left[e^{-4 i t A_{r}^{\dagger}} e^{2 x A_{r}^{\dagger}}-Q_{r, \Sigma} e^{-4 i t A_{r}^{2}} e^{-2 x A_{r}} N_{r, \Omega}\right]^{-1} \times \\
& \times e^{-(y-x) A_{r}^{\dagger}} C_{r}^{\dagger} \Sigma^{-1} \\
K^{\mathrm{dn}}(x, y, t) & =C_{r}\left[e^{4 i t A_{r}^{2}} e^{2 x A_{r}}-N_{r, \Omega} e^{4 i t A_{r}^{\dagger}{ }^{2}} e^{-2 x A_{r}^{\dagger}} Q_{r, \Sigma}\right]^{-1} \times \\
& \times N_{r, \Omega} e^{4 i t A_{r}^{\dagger}} e^{-(x+y) A_{r}^{\dagger}} C_{r}^{\dagger} \Sigma^{-1},
\end{aligned}
$$

as well as

$$
\begin{aligned}
L^{\mathrm{up}}(x, y, t) & =C_{l}\left[e^{-4 i t A_{l}^{2}} e^{-2 x A_{l}}-N_{l, \Omega} e^{-4 i t A_{l}^{\dagger}} e^{2 x A_{l}^{\dagger}} Q_{l, \Sigma}\right]^{-1} \times \\
& \times N_{l, \Omega} e^{-4 i t A_{l}^{\dagger^{2}}} e^{(x+y) A_{l}^{\dagger}} C_{l}^{\dagger} \Sigma^{-1} \\
L^{\mathrm{dn}}(x, y, t) & =-\Omega^{-1} B_{l}^{\dagger}\left[e^{4 i t A_{l}^{\dagger}} e^{-2 x A_{l}^{\dagger}}-Q_{l, \Sigma} e^{4 i t A_{l}^{2}} e^{2 x A_{l}} N_{l, \Omega}\right]^{-1} \times \\
& \times e^{-(x-y) A_{l}^{\dagger}} C_{l}^{\dagger} \Sigma^{-1}, \\
\bar{L}^{\mathrm{up}}(x, y, t) & =-C_{l}\left[e^{-4 i t A_{l}^{2}} e^{-2 x A_{l}}-N_{l, \Omega} e^{-4 i t A_{l}^{\dagger^{2}}} e^{2 x A_{l}^{\dagger}} Q_{l, \Sigma}\right]^{-1} \times \\
& \times e^{-(x-y) A_{l}} B_{l}, \\
\bar{L}^{\mathrm{dn}}(x, y, t) & =\Omega^{-1} B_{l}^{\dagger}\left[e^{4 i t A_{l}^{\dagger}} e^{-2 x A_{l}^{\dagger}}-Q_{l, \Sigma} e^{4 i t A_{l}^{2}} e^{2 x A_{l}} N_{l, \Omega}\right]^{-1} \times \\
& \times Q_{l, \Sigma} e^{4 i t A_{l}^{2}} e^{(x+y) A_{l}} B_{l} .
\end{aligned}
$$

We observe that the expressions (4.15) and (4.16) do not require the minimality of any matrix triplets. 
Let us now use (2.8) in (4.15) and (4.16). We get

$$
\begin{aligned}
& Q(x, t)=2 \Omega^{-1} B_{r}^{\dagger}\left[e^{2 x A_{r}^{\dagger}} e^{-4 i t A_{r}^{\dagger 2}}-Q_{r, \Sigma} e^{-2 x A_{r}} e^{-4 i t A_{r}^{2}} N_{r, \Omega}\right]^{-1} C_{r}^{\dagger} \Sigma^{-1}, \\
& Q(x, t)=-2 C_{l}\left[e^{-2 x A_{l}} e^{-4 i t A_{l}^{2}}-N_{l, \Omega} e^{2 x A_{l}^{\dagger}} e^{-4 i t A_{l}^{\dagger 2}} Q_{l, \Sigma}\right]^{-1} B_{l}, \\
& R(x, t)=2 C_{r}\left[e^{2 x A_{r}} e^{4 i t A_{r}^{2}}-N_{r, \Omega} e^{-2 x A_{r}^{\dagger}} e^{4 i t A_{r}^{\dagger}} Q_{r, \Sigma}\right]^{-1} B_{r}, \\
& R(x, t)=-2 \Omega^{-1} B_{l}^{\dagger}\left[e^{-2 x A_{l}^{\dagger}} e^{4 i t A_{l}^{\dagger^{2}}}-Q_{l, \Sigma} e^{2 x A_{l}} e^{4 i t A_{l}^{2}} N_{l, \Omega}\right]^{-1} C_{l}^{\dagger} \Sigma^{-1} .
\end{aligned}
$$

Observe that the symmetry relation $R=\Sigma Q^{\dagger} \Omega$ is satisfied.

\subsection{Jost solutions and scattering coefficients}

The modified Jost solutions are as follows:

$$
\begin{aligned}
\bar{N}^{\mathrm{up}}(x, t, k) & =I_{2}-i \Omega^{-1} B_{r}^{\dagger}\left[e^{-4 i t A_{r}^{\dagger 2}} e^{2 x A_{r}^{\dagger}}-Q_{r, \Sigma} e^{-4 i t A_{r}^{2}} e^{-2 x A_{r}} N_{r, \Omega}\right]^{-1} \times \\
& \times Q_{r, \Sigma} e^{-4 i t A_{r}^{2}} e^{-2 x A_{r}}\left(k I_{p}-i A_{r}\right)^{-1} B_{r}, \\
\bar{N}^{\mathrm{dn}}(x, t, k) & =i C_{r}\left[e^{4 i t A_{r}^{2}} e^{2 x A_{r}}-N_{r, \Omega} e^{4 i t A_{r}^{\dagger}} e^{-2 x A_{r}^{\dagger}} Q_{r, \Sigma}\right]^{-1} \times \\
& \times\left(k I_{p}-A_{r}\right)^{-1} B_{r}, \\
N^{\mathrm{up}}(x, t, k) & =-i \Omega^{-1} B_{r}^{\dagger}\left[e^{-4 i t A_{r}^{\dagger 2}} e^{2 x A_{r}^{\dagger}}-Q_{r, \Sigma} e^{-4 i t A_{r}^{2}} e^{-2 x A_{r}} N_{r, \Omega}\right]^{-1} \times \\
& \times\left(k I_{p}+i A_{r}^{\dagger}\right)^{-1} C_{r}^{\dagger} \Sigma^{-1} \\
N^{\mathrm{dn}}(x, t, k) & =I_{2}+i C_{r}\left[e^{4 i t A_{r}^{2}} e^{2 x A_{r}}-N_{r, \Omega} e^{4 i t A_{r}^{\dagger}} e^{-2 x A_{r}^{\dagger}} Q_{r, \Sigma}\right]^{-1} \times \\
& \times N_{r, \Omega} e^{4 i t A_{r}^{\dagger^{2}}} e^{-2 x A_{r}^{\dagger}}\left(k I_{p}+i A_{r}^{\dagger}\right)^{-1} C_{r}^{\dagger} \Sigma^{-1}
\end{aligned}
$$

as well as

$$
\begin{aligned}
M^{\text {up }}(x, t, k) & =I_{2}+i C_{l}\left[e^{-4 i t A_{l}^{2}} e^{-2 x A_{l}}-N_{l, \Omega} e^{-4 i t A_{l}^{\dagger^{2}}} e^{2 x A_{l}^{\dagger}} Q_{l, \Sigma}\right]^{-1} \times \\
& \times N_{l, \Omega} e^{-4 i t A_{l}^{\dagger}} e^{2 x A_{l}^{\dagger}}\left(k I_{p}+i A_{l}^{\dagger}\right)^{-1} C_{l}^{\dagger} \Sigma^{-1} \\
M^{\mathrm{dn}}(x, t, k) & =-i \Omega^{-1} B_{l}^{\dagger}\left[e^{4 i t A_{l}^{\dagger}} e^{-2 x A_{l}^{\dagger}}-Q_{l, \Sigma} e^{4 i t A_{l}^{2}} e^{2 x A_{l}} N_{l, \Omega}\right]^{-1} \times \\
& \times\left(k I_{p}+i A_{l}^{\dagger}\right)^{-1} C_{l}^{\dagger} \Sigma^{-1},
\end{aligned}
$$




$$
\begin{aligned}
\bar{M}^{\mathrm{up}}(x, t, k) & =i C_{l}\left[e^{-4 i t A_{l}^{2}} e^{-2 x A_{l}}-N_{l, \Omega} e^{-4 i t A_{l}^{\dagger 2}} e^{2 x A_{l}^{\dagger}} Q_{l, \Sigma}\right]^{-1} \times \\
& \times\left(k I_{p}-i A_{l}\right)^{-1} B_{l}, \\
\bar{M}^{\mathrm{dn}}(x, t, k) & =I_{2}-i \Omega^{-1} B_{l}^{\dagger}\left[e^{4 i t A_{l}^{\dagger}} e^{-2 x A_{l}^{\dagger}}-Q_{l, \Sigma} e^{4 i t A_{l}^{2}} e^{2 x A_{l}} N_{l, \Omega}\right]^{-1} \times \\
& \times Q_{l, \Sigma} e^{4 i t A_{l}^{2}} e^{2 x A_{l}}\left(k I_{p}-i A_{l}\right)^{-1} B_{l} .
\end{aligned}
$$

Taking suitable limits as $x \rightarrow \pm \infty$, we obtain the scattering coefficients

$$
\begin{aligned}
& \bar{a}(k)=I_{2}+i \Omega^{-1} B_{r}^{\dagger} N_{r, \Omega}^{-1}\left(k I_{p}-i A_{r}\right)^{-1} B_{r}, \\
& a(k)=I_{2}-i C_{r} Q_{r, \Sigma}^{-1}\left(k I_{p}+i A_{r}^{\dagger}\right)^{-1} C_{r}^{\dagger} \Sigma^{-1}, \\
& c(k)=I_{2}-i C_{l} Q_{l, \Sigma}^{-1}\left(k I_{p}+i A_{l}^{\dagger}\right)^{-1} C_{l}^{\dagger} \Sigma^{-1}, \\
& \bar{c}(k)=I_{2}+i \Omega^{-1} B_{l}^{\dagger} N_{l, \Omega}^{-1}\left(k I_{p}-i A_{l}\right)^{-1} B_{l},
\end{aligned}
$$

provided the matrices $Q_{r, \Sigma}, N_{r, \Omega}, Q_{l, \Sigma}$, and $N_{l, \Omega}$ are invertible. It is easily verified that their invertibility implies that the matrix triplets $\left(A_{r}, B_{r}, C_{r}\right)$ and $\left(A_{l}, B_{l}, C_{l}\right)$ are minimal. Using the Lyapunov equations we obtain the inverted scattering coefficients

$$
\begin{aligned}
& \bar{a}(k)^{-1}=I_{2}-i \Omega^{-1} B_{r}^{\dagger}\left(k I_{p}+i A_{r}^{\dagger}\right)^{-1} N_{r, \Omega}^{-1} B_{r}, \\
& a(k)^{-1}=I_{2}+i C_{r}\left(k I_{p}-i A_{r}\right)^{-1} Q_{r, \Sigma}^{-1} C_{r}^{\dagger} \Sigma^{-1}, \\
& c(k)^{-1}=I_{2}+i C_{l}\left(k I_{p}-i A_{l}\right)^{-1} Q_{l, \Sigma}^{-1} C_{l}^{\dagger} \Sigma^{-1}, \\
& \bar{c}(k)^{-1}=I_{2}-i \Omega^{-1} B_{l}^{\dagger}\left(k I_{p}+i A_{l}^{\dagger}\right)^{-1} N_{l, \Omega}^{-1} B_{l} .
\end{aligned}
$$

So far we have not used the complex symmetry of the potential $Q(x, t)$ to derive symmetry relations for the matrix triplets $\left(A_{r}, B_{r}, C_{r}\right)$ and $\left(A_{l}, B_{l}, C_{l}\right)$. This we intend to remedy presently. Starting from the Marchenko integral kernel representations (3.8) with zero reflection coefficient terms, under the condition of minimality of the triplets there exists, for (3.8a), a unique invertible $p \times p$ matrix $S$ such that

$$
A_{r} S=S A_{r}^{T}, \quad S B_{r}=C_{r}^{T}, \quad B_{r}^{T}=C_{r} S^{-1} .
$$

Taking transposes, we obtain the similarity relations

$$
A_{r} S^{T}=S^{T} A_{r}^{T}, \quad S^{T} B_{r}=C_{r}^{T}, \quad B_{r}^{T}=C_{r} S^{T^{-1}} .
$$

Using the uniqueness of the similarity, we get $S^{T}=S$. In other words, $S$ is a complex symmetric matrix. If the similarity $S$ in (4.23) is the identity 
matrix, then $A_{r}$ is a complex symmetric matrix and $B_{r}$ is the transpose of $C_{r}$, though it might not be immediate how to relate the matrix triplet to the norming constants as defined in [22]. The same conclusions can be drawn for the other three matrix triplet representations whenever minimal.

\subsection{Four cases of the matrix triplet method}

In case 1 (defocusing case, $\Sigma=\Omega=I_{2}$ ) there do not exist any multisoliton solutions.

In case 2 (focusing case, $\Sigma=-\Omega=I_{2}$ ) the matrices $Q_{r, \Sigma},-N_{r, \Omega}, Q_{l, \Sigma}$, and $-N_{l, \Omega}$ are positive selfadjoint and hence nonsingular $[24,6]$ iff the corresponding matrix triplet is minimal. This implies that the four matrices in (4.8) have a positive determinant for each $x \in \mathbb{R}$. Thus in case 2 any matrix triplet, minimal or not, leads to a multisoliton solution without finite singularities which is exponentially decaying as $x \rightarrow \pm \infty$ (see $[6,24]$ ).

In cases 3 and 4 it is possible for the matrix triplet $\left(A_{r}, B_{r}, C_{r}\right)$ to be minimal, whereas one (or both) of the matrices $Q_{r, \Sigma}$ and $N_{r, \Omega}$ is singular. In this situation we may find reflectionless NLS solutions that decay exponentially as $x \rightarrow+\infty$ but do not decay as $x \rightarrow-\infty$. We refer to [22, Eq. (84)] for an example of this situation. In either case it is also possible that, for some instant $t$, one (or both) of the matrices $\Gamma_{r}(x, t)$ and $\bar{\Gamma}_{r}(x, t)$ is singular at certain finite $x$-values. In this situation the reflectionless solution has a pole at some finite $x$ for this particular instant $t$ and does not represent a soliton solution.

Let us illustrate these phenomena using well-known results for one-soliton solutions with norming constant $C_{1}=\left(\begin{array}{cc}c_{1} & c_{0} \\ c_{0} & c_{-1}\end{array}\right)$. As shown in [22, Sec. 4], a one-soliton solution (without singularities) occurs under the necessary and sufficient condition that

$$
\begin{cases}\left|c_{1}\right|^{2}+\left|c_{-1}\right|^{2}-2\left|c_{0}\right|^{2}<2\left|c_{1} c_{-1}-c_{0}^{2}\right|, & \operatorname{det} C_{1} \neq 0 \\ \left|c_{1}\right|=\left|c_{-1}\right| \text { and } c_{0}^{2}=c_{1} c_{-1}, & \operatorname{det} C_{1}=0\end{cases}
$$

in Case 3 and

$$
\begin{cases}\left|c_{1}\right|^{2}+\left|c_{-1}\right|^{2}-2\left|c_{0}\right|^{2}>-2\left|c_{1} c_{-1}-c_{0}^{2}\right|, & \operatorname{det} C_{1} \neq 0, \\ \text { any singular matrix } C_{1}, & \operatorname{det} C_{1}=0,\end{cases}
$$

in Case 4. Thus there do not exist norming constants $C_{1}$ that do not lead to a one-soliton solution in Case 3 nor to a one-soliton solution in Case 4. 


\section{Substitution in the matrix NLS equation}

In this section we prove, by direct substitution, that the potentials $Q(x, t)$ in $(4.17 \mathrm{~b})$ and $R(x, t)$ in $(4.17 \mathrm{~d})$ satisfy the NLS equation (1.1). A similar substitution proved the potentials $Q(x, t)$ and $R(x, t)$ in (4.19a) and (4.19c) to satisfy the NLS equation [4].

Let us write

$$
Q(x, t)=-2 C_{l} e^{x A_{l}} \boldsymbol{\Gamma}_{l}(x, t)^{-1} e^{4 i t A_{l}^{2}} e^{x A_{l}} B_{l},
$$

where

$$
\boldsymbol{\Gamma}_{l}(x, t)=I_{p}-e^{4 i t A_{l}^{2}} e^{x A_{l}} N_{l, \Omega} e^{-4 i t A_{l}^{\dagger}} e^{2 x A_{l}^{\dagger}} Q_{l, \Sigma} e^{x A_{l}},
$$

$p$ being the matrix order of $A_{l}$. Then we can write $\boldsymbol{\Gamma}_{l}(x, t)$ as follows

$$
\Gamma_{l}(x, t)=I_{p}-\mathcal{N}(x, t) \mathcal{Q}(x),
$$

where

$$
\mathcal{N}(x, t)=e^{4 i t A_{l}^{2}} e^{x A_{l}} N_{l, \Omega} e^{-4 i t A_{l}^{\dagger^{2}}} e^{x A_{l}^{\dagger}}, \quad \mathcal{Q}(x)=e^{x A_{l}^{\dagger}} Q_{l, \Sigma} e^{x A_{l}} .
$$

It is then easily verified that

$$
\begin{aligned}
\mathcal{N}_{x} & =A_{l} \mathcal{N}+\mathcal{N} A_{l}^{\dagger}, \\
\mathcal{Q}_{x} & =A_{l}^{\dagger} \mathcal{Q}+\mathcal{Q} A_{l}, \\
\mathcal{N}_{t} & =4 i\left[A_{l}^{2} \mathcal{N}-\mathcal{N} A_{l}^{\dagger^{2}}\right] \\
\left(\boldsymbol{\Gamma}_{l}^{-1}\right)_{x} & =-\boldsymbol{\Gamma}_{l}^{-1}\left(\boldsymbol{\Gamma}_{l}\right)_{x} \boldsymbol{\Gamma}_{l}^{-1} \\
& =2 \boldsymbol{\Gamma}_{l}^{-1}\left(A_{l}+\mathcal{N} A_{l}^{\dagger} \mathcal{Q}\right) \boldsymbol{\Gamma}_{l}^{-1}-\boldsymbol{\Gamma}_{l}^{-1} A_{l}-A_{l} \boldsymbol{\Gamma}_{l}^{-1} \\
\left(\boldsymbol{\Gamma}_{l}^{-1}\right)_{t} & =-\boldsymbol{\Gamma}_{l}^{-1}\left(\boldsymbol{\Gamma}_{l}\right)_{t} \boldsymbol{\Gamma}_{l}^{-1}=4 i \boldsymbol{\Gamma}_{l}^{-1}\left(A_{l}^{2} \boldsymbol{\Gamma}_{l}-A_{l}^{2}+\mathcal{N}\left(A_{l}^{\dagger}\right)^{2} \mathcal{Q}\right) \boldsymbol{\Gamma}_{l}^{-1}
\end{aligned}
$$

Then we readily differentiate (5.1) with respect to $x$ to get

$$
\begin{aligned}
Q_{x} & =-2 C_{l} e^{x A_{l}}\left[\boldsymbol{\Gamma}_{l}^{-1} A_{l}+A_{l} \boldsymbol{\Gamma}_{l}^{-1}-\boldsymbol{\Gamma}_{l}^{-1}\left(\boldsymbol{\Gamma}_{l}\right)_{x} \boldsymbol{\Gamma}_{l}^{-1}\right] e^{4 i t A_{l}^{2}} e^{x A_{l}} B_{l} \\
& =-4 C_{l} e^{x A_{l}} \boldsymbol{\Gamma}_{l}^{-1}\left[A_{l}+\mathcal{N} A_{l}^{\dagger} \mathcal{Q}\right] \boldsymbol{\Gamma}_{l}^{-1} e^{4 i t A_{l}^{2}} e^{x A_{l}} B_{l},
\end{aligned}
$$

where we have used (5.4a), (5.4b), and (5.4d). 
Let us now substitute $Q$ and $R$ in (4.17b) and (4.17d) into (1.1). Differentiating (5.1) and taking into account that $\Gamma_{l}+\mathcal{N} \mathcal{Q}=I_{p}$ and equation (5.4c), we obtain

$$
\begin{aligned}
i Q_{t} & =8 C_{l} e^{x A_{l}} \boldsymbol{\Gamma}_{l}^{-1}\left[A_{l}^{2} \boldsymbol{\Gamma}_{l}+A_{l}^{2} \mathcal{N} \mathcal{Q}-\mathcal{N} A_{l}^{\dagger^{2}} \mathcal{Q}\right] \boldsymbol{\Gamma}_{l}^{-1} e^{4 i t A_{l}^{2}} e^{x A_{l}} B_{l} \\
& =8 C_{l} e^{x A_{l}} \boldsymbol{\Gamma}_{l}^{-1}\left[A_{l}^{2}-\mathcal{N} A_{l}^{\dagger} \mathcal{Q}\right] \boldsymbol{\Gamma}_{l}^{-1} e^{4 i t A_{l}^{2}} e^{x A_{l}} B_{l} .
\end{aligned}
$$

Using

$$
\left(A_{l}+\mathcal{N} A_{l}^{\dagger} \mathcal{Q}\right)_{x}=A_{l} \mathcal{N} A_{l}^{\dagger} \mathcal{Q}+\mathcal{N} A_{l}^{\dagger} \mathcal{Q} A_{l}+2 \mathcal{N} A_{l}^{\dagger} \mathcal{Q}
$$

we obtain

$$
\begin{aligned}
Q_{x x} & =-4 C_{l} e^{x A_{l}}\left\{A_{l} \boldsymbol{\Gamma}_{l}^{-1}\left(A_{l}+\mathcal{N} A_{l}^{\dagger} \mathcal{Q}\right) \boldsymbol{\Gamma}_{l}^{-1}+\boldsymbol{\Gamma}_{l}^{-1}\left(A_{l}+\mathcal{N} A_{l}^{\dagger} \mathcal{Q}\right) \boldsymbol{\Gamma}_{l}^{-1} A_{l}\right\} e^{4 i t A_{l}^{2}} e^{x A_{l}} B_{l} \\
& -4 C_{l} e^{x A_{l}} \boldsymbol{\Gamma}_{l}^{-1}\left\{A_{l} \mathcal{N} A_{l}^{\dagger} \mathcal{Q}+\mathcal{N} A_{l}^{\dagger} \mathcal{Q} A_{l}+2 \mathcal{N} A_{l}^{\dagger} \mathcal{Q}\right\} \boldsymbol{\Gamma}_{l}^{-1} e^{4 i t A_{l}^{2}} e^{x A_{l}} B_{l} \\
& -4 C_{l} e^{x A_{l}}\left[2 \boldsymbol{\Gamma}_{l}^{-1}\left(A_{l}+\mathcal{N} A_{l}^{\dagger} \mathcal{Q}\right) \boldsymbol{\Gamma}_{l}^{-1}-\boldsymbol{\Gamma}_{l}^{-1} A_{l}-A_{l} \boldsymbol{\Gamma}_{l}^{-1}\right]\left(A_{l}+\mathcal{N} A_{l}^{\dagger} \mathcal{Q}\right) \boldsymbol{\Gamma}_{l}^{-1} e^{4 i t A_{l}^{2}} e^{x A_{l}} B_{l} \\
& -4 C_{l} e^{x A_{l}} \boldsymbol{\Gamma}_{l}^{-1}\left(A_{l}+\mathcal{N} A_{l}^{\dagger} \mathcal{Q}\right)\left[2 \boldsymbol{\Gamma}_{l}^{-1}\left(A_{l}+\mathcal{N} A_{l}^{\dagger} \mathcal{Q}\right) \boldsymbol{\Gamma}_{l}^{-1}-\boldsymbol{\Gamma}_{l}^{-1} A_{l}-A_{l} \boldsymbol{\Gamma}_{l}^{-1}\right] e^{4 i t A_{l}^{2}} e^{x A_{l}} B_{l} \\
& =8 C_{l} e^{x A_{l}} \boldsymbol{\Gamma}_{l}^{-1}\left(A_{l}^{2}-\mathcal{N} A_{l}^{\dagger} \mathcal{Q}\right) \boldsymbol{\Gamma}_{l}^{-1} e^{4 i t A_{l}^{2}} e^{x A_{l}} B_{l} \\
& -16 C_{l} e^{x A_{l}} \boldsymbol{\Gamma}_{l}^{-1}\left(A_{l}+\mathcal{N} A_{l}^{\dagger} \mathcal{Q}\right) \boldsymbol{\Gamma}_{l}^{-1}\left(A_{l}+\mathcal{N} A_{l}^{\dagger} \mathcal{Q}\right) \boldsymbol{\Gamma}_{l}^{-1} e^{4 i t A_{l}^{2}} e^{x A_{l}} B_{l},
\end{aligned}
$$

where we have used (5.3). Finally, using that

$$
\begin{aligned}
A_{l} \mathcal{N}+\mathcal{N} A_{l}^{\dagger} & =e^{4 i t A_{l}^{2}} e^{x A_{l}} B_{l} \Omega^{-1} B_{l}^{\dagger} e^{-4 i t A_{l}^{\dagger^{2}}} e^{x A_{l}^{\dagger}}, \\
A_{l}^{\dagger} \mathcal{Q}+\mathcal{Q} A_{l} & =e^{x A_{l}^{\dagger}} C_{l}^{\dagger} \Sigma^{-1} C e^{x A_{l}},
\end{aligned}
$$

we get

$$
\begin{aligned}
-2 Q R Q & =16 C_{l} e^{x A_{l}} \boldsymbol{\Gamma}_{l}^{-1}\left(A_{l} \mathcal{N}+\mathcal{N} A_{l}^{\dagger}\right) \boldsymbol{\Gamma}_{l}^{\dagger^{-1}}\left(A_{l}^{\dagger} \mathcal{Q}+\mathcal{Q} A_{l}\right) \boldsymbol{\Gamma}_{l}^{-1} e^{4 i t A_{l}^{2}} e^{x A_{l}} B_{l} \\
& =-16 C_{l} e^{x A_{l}} \boldsymbol{\Gamma}_{l}^{-1}\left[A_{l}^{2}-\mathcal{N} A_{l}^{\dagger} \mathcal{Q}\right] \boldsymbol{\Gamma}_{l}^{-1} e^{4 i t A_{l}^{2}} e^{x A_{l}} B_{l} \\
& +16 C_{l} e^{x A_{l}} \boldsymbol{\Gamma}_{l}^{-1}\left(A_{l}+\mathcal{N} A_{l}^{\dagger} \mathcal{Q}\right) \boldsymbol{\Gamma}_{l}^{-1}\left(A_{l}+\mathcal{N} A_{l}^{\dagger} \mathcal{Q}\right) \boldsymbol{\Gamma}_{l}^{-1} e^{4 i t A_{l}^{2}} e^{x A_{l}} B_{l} .
\end{aligned}
$$

Since the final expressions in (5.6), (5.7), and (5.8) add up to zero, the potential $Q(x, t)$ satisfies the matrix NLS equation (1.1). 


\section{Examples of reflectionless NLS solutions}

In this section we compute the reflectionless NLS solutions explicitly for certain elementary matrix triplets based on the Jordan normal form. We also give necessary and sufficient condition for the minimality of these triplets.

It is well known that it is possible to choose the triplet $\left(A_{l}, B_{l}, C_{l}\right)$ in a "canonical way." Following the same arguments used in [4], for some appropriate positive integer $r$, we have

$$
\begin{aligned}
A_{l} & =\left(\begin{array}{cccc}
A_{1} & 0 & \ldots & 0 \\
0 & A_{2} & \ldots & 0 \\
\vdots & \vdots & \ddots & \vdots \\
0 & 0 & \ldots & A_{r}
\end{array}\right), \\
B_{l} & =\left(\begin{array}{c}
B_{1} \\
\vdots \\
B_{r}
\end{array}\right), \quad C_{l}=\left(\begin{array}{lll}
C_{1} & \ldots & C_{r}
\end{array}\right)
\end{aligned}
$$

where in the case of an eigenvalue $\lambda_{j}$ of $i A_{j}$ with positive imaginary part the corresponding blocks are given by

$$
A_{j}:=\left(\begin{array}{cccccc}
-i \lambda_{j} I_{2} & -I_{2} & 0 & \ldots & 0 & 0 \\
0 & -i \lambda_{j} I_{2} & -I_{2} & \ldots & 0 & 0 \\
0 & 0 & -i \lambda_{j} I_{2} & \ldots & 0 & 0 \\
\vdots & \vdots & \vdots & \ddots & \vdots & \vdots \\
0 & 0 & 0 & \ldots & -i \lambda_{j} I_{2} & -I_{2} \\
0 & 0 & 0 & \ldots & 0 & -i \lambda_{j} I_{2}
\end{array}\right) .
$$

Here $A_{j}$ has size $2 n_{j} \times 2 n_{j}, B_{l}$ has size $2 n_{j} \times 2$, and $C_{l}$ has size $2 \times 2 n_{j}$. Observe that $2\left(n_{1}+\ldots+n_{r}\right)=p$.

To arrive at a reflectionless solution formula for a complex symmetric potential $Q(x, t)$, we let $E_{n}$ be the $n \times n$ matrix with $I_{2}$ as its entries on the trailing diagonal and $0_{2 \times 2}$ as its other entries. In the matrix triplet (6.1) the similarity transformation $S$ as in (6.2) below making $A_{l}^{T}$ similar to $A_{l}$ is the direct sum

$$
S=E_{n_{1}} \oplus \ldots \oplus E_{n_{r}}
$$


We then define $B_{l}$ in terms of $C_{l}$ by $B_{l}=S C_{l}^{T}$ or

$$
B_{l}=\left(\begin{array}{c}
E_{n_{1}} C_{1}^{T} \\
E_{n_{2}} C_{2}^{T} \\
\vdots \\
E_{n_{r}} C_{r}^{T}
\end{array}\right),
$$

where we note that premultiplication of a block matrix with $2 \times 2$ entries having $n$ rows by $E_{n}$ leads to a reversal of the order of the rows in this matrix. If $A_{l}$ is a diagonal matrix with $2 \times 2$ diagonal entries, we have $S=I_{p}$ and $B_{l}=C_{l}^{T}$. Unfortunately, in this way it is not easy to relate the matrix triplet to the norming constants as defined in [22].

To define a minimal triplet for a complex symmetric potential whose entries are easily related to the norming constants, we now consider the matrix triplet $\left(A_{l}, B_{l}, C_{l}\right)$, where

$$
A_{l}=\operatorname{diag}\left(-i \lambda_{1} I_{2}, \ldots,-i \lambda_{r} I_{2}\right)
$$

for distinct algebraically simple eigenvalues $\lambda_{1}, \ldots, \lambda_{r} \in \mathbb{C}^{+}$and

$$
B_{l}=\left(\begin{array}{c}
I_{2} \\
\vdots \\
I_{2}
\end{array}\right), \quad C_{l}=-i\left(\begin{array}{lll}
C_{1} & \ldots & C_{r}
\end{array}\right)
$$

Then the identities (4.22) (with the subscripts $r$ replaced by $l$ ) imply that $S=-i \operatorname{diag}\left(C_{1}^{T}, \ldots, C_{r}^{T}\right)$ for suitable invertible $2 \times 2$ matrices $C_{1}, \ldots, C_{r}$. Thus if we take the complex symmetric matrices $C_{1}, \ldots, C_{r}$ as the norming constants, then the triplet $\left(A_{l}, B_{l}, C_{l}\right)$ is minimal iff none of the norming constants is a singular matrix.

Example 6.1 (One-soliton solution) Consider the matrix triplet

$$
A_{l}=(\eta-i \xi) I_{2}, \quad B_{l}=\Omega, \quad C_{l}=-i C_{1} \Omega^{-1},
$$

where $\xi \in \mathbb{R}, \eta>0$, and $C_{1}$ is a complex symmetric $2 \times 2$ matrix. Then (cf. $[22, \mathrm{Eq} \cdot(71)])$

$$
\begin{aligned}
Q(x, t) & =2 i e^{-2 \eta(x+4 \xi t)} e^{-4 i t\left(\xi^{2}-\eta^{2}\right)} e^{-2 i \xi x} \times \\
& \times\left[I_{2}-\frac{e^{-4 \eta(x+4 \xi t)}}{4 \eta^{2}} \Omega^{-1} C_{1}^{\dagger} \Sigma^{-1} C_{1}\right]^{-1} \Omega^{-1} C_{1}^{\dagger} \Sigma^{-1}
\end{aligned}
$$


is a one-soliton solution. The triplet is minimal iff $\operatorname{det} C_{1}$ is nonzero. It is easily verified that

$\bar{N}^{\mathrm{up}}(x, t, k)=I_{2}-\frac{i e^{-4 \eta(x+4 \xi t)}}{2 \eta[k-\xi-i \eta]}\left[I_{2}-\frac{e^{-4 \eta(x+4 \xi t)}}{4 \eta^{2}} \Omega^{-1} C_{1}^{\dagger} \Sigma^{-} C_{1}\right]^{-1} \Omega^{-1} C_{1}^{\dagger} \Sigma^{-1}$,

so that

$$
\bar{N}^{\mathrm{up}}(x, t, \xi-i \eta)=\left[I_{2}-\frac{e^{-4 \eta(x+4 \xi t)}}{4 \eta^{2}} \Omega^{-1} C_{1}^{\dagger} \Sigma^{-1} C_{1}\right]^{-1}
$$

Example 6.2 (Two-soliton solutions) We plot (figures 1, 2 and 3)) the three distinct elements of the complex symmetric matrix $Q(x, t)$ for the same triplet $\left(A_{r}, B_{r}, C_{r}\right)$ in the cases 2,3 and 4 , where

$$
\begin{gathered}
A_{r}=\left(\begin{array}{llll}
3 & 0 & 0 & 0 \\
0 & 3 & 0 & 0 \\
0 & 0 & 2 & 0 \\
0 & 0 & 0 & 2
\end{array}\right), \\
C_{r}=-i\left(\begin{array}{llll}
1 & 2 & 1 & 0 \\
2 & 3 & 0 & 1
\end{array}\right), \quad B_{r}=\left(\begin{array}{ll}
1 & 0 \\
0 & 1 \\
1 & 0 \\
0 & 1
\end{array}\right) .
\end{gathered}
$$

We observe that the plots of case 4 is similar to the plots of the focusing case (case 2). Instead, in case 3, the plots show that a singularity arises in the following matrix (which appears in the expression of the soliton solution $(4.17 \mathrm{a}))$

$$
\left[e^{2 x A_{r}^{\dagger}} e^{-4 i t A_{r}^{\dagger}}-Q_{r, \Sigma} e^{-2 x A_{r}} e^{-4 i t A_{r}^{2}} N_{r, \Omega}\right] .
$$

This fact is not surprising because the case 3 is closed to the defocusing case where, as underlined in the previous section, no soliton solutions exists.

Note that $C_{r}$ consists of two nonsingular block elements. We have not found necessary and sufficient conditions on the two discrete eigenvalues and the associated norming constants in order that, in Case 3 or in Case 4, there are no finite singularities.

Let us now consider the matrix triplet $\left(A_{r}, B_{r}, C_{r}\right)$ as in (6.1) for distinct (not necessarily algebraically simple) eigenvalues $\lambda_{1}, \ldots, \lambda_{r} \in \mathbb{C}^{+}$. Then the 

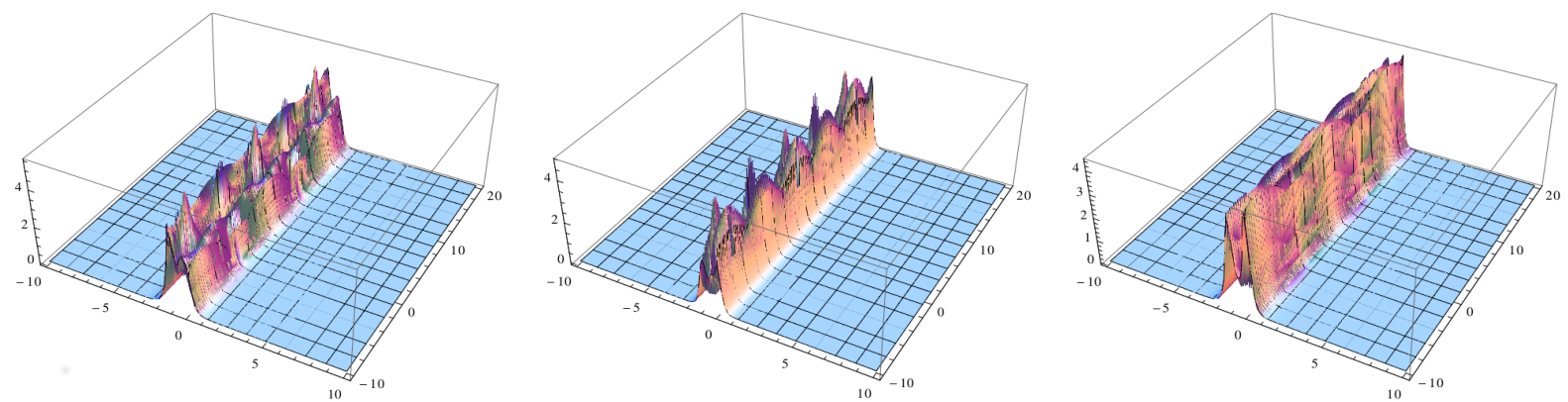

Figure 1: An example of a two soliton solution in the focusing case.
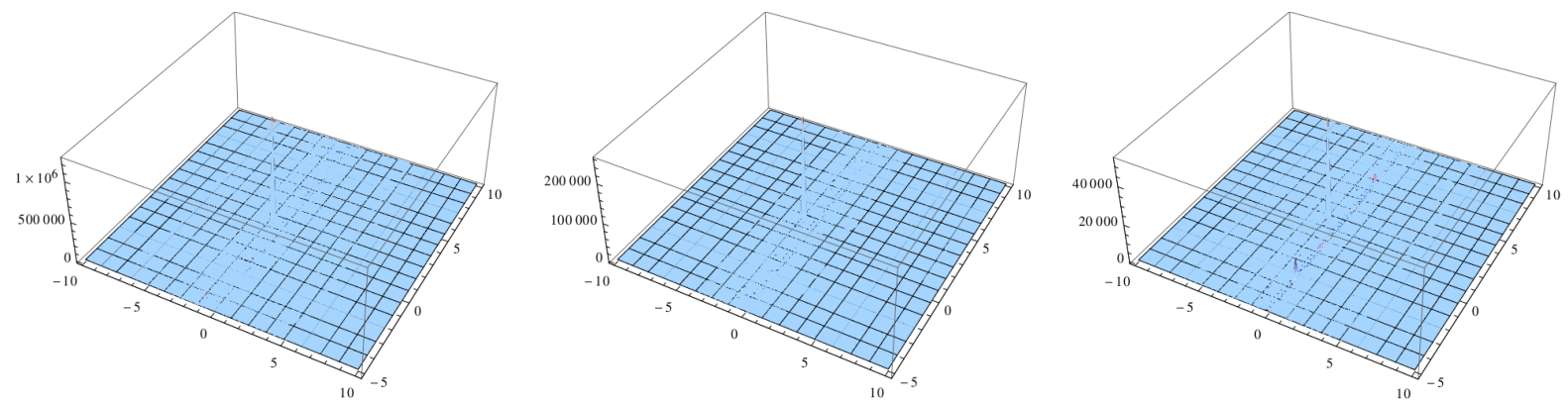

Figure 2: An example of a two soliton solution in case 3. Note the presence of a finite singularity.
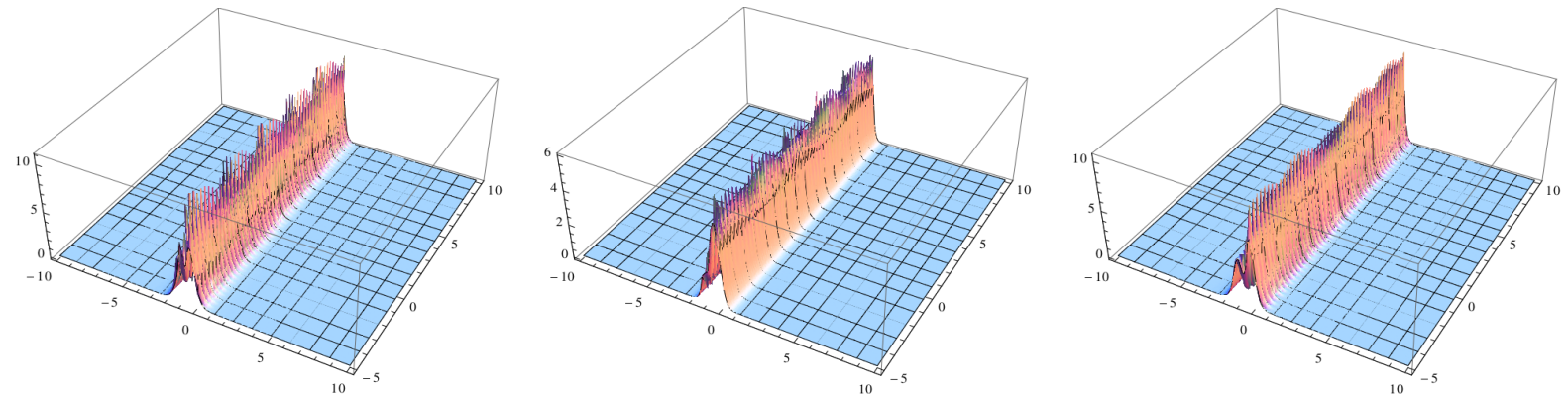

Figure 3: An example of a two soliton solution in case 4. 
first of the identities (4.22) imply that $S=\operatorname{diag}\left(S_{1}, \ldots, S_{r}\right)$, where $S_{j}$ has size $2 n_{j} \times 2 n_{j}$ and has the block Hankel form

$$
S_{j}=\left(\begin{array}{cccccc}
S_{1 ; j} & S_{2 ; j} & S_{3 ; j} & \ldots & \ldots & S_{n_{j} ; j} \\
S_{2 ; j} & S_{3 ; j} & S_{4 ; j} & \ldots & S_{n_{j} ; j} & 0_{2 \times 2} \\
S_{3 ; j} & S_{4 ; j} & S_{5 ; j} & \ldots & 0_{2 \times 2} & 0_{2 \times 2} \\
\vdots & & & & 0_{2 \times 2} & 0_{2 \times 2} \\
\vdots & S_{n_{j} ; j} & 0_{2 \times 2} & \ldots & 0_{2 \times 2} & 0_{2 \times 2} \\
S_{n_{j} ; j} & 0_{2 \times 2} & \ldots & \ldots & 0_{2 \times 2} & 0_{2 \times 2}
\end{array}\right) .
$$

Then $S$ is invertible iff all matrices $S_{n_{j} ; j}$ are invertible. If we then choose

$$
C_{j}=-i\left(\begin{array}{lllll}
i^{n_{j}-1} C_{1 ; j} & i^{n_{j}-2} C_{2 ; j} & \ldots & i C_{n_{j}-1 ; j} & C_{n_{j} ; j}
\end{array}\right)
$$

and $B_{j}^{T}=\left(\begin{array}{llll}I_{2} & 0_{2 \times 2} & \ldots & 0_{2 \times 2}\end{array}\right)$, then $S_{j ; l}=i^{n_{l}-j-1} C_{j ; l}^{T}\left(l=1,2, \ldots, n_{j}\right)$. In other words, the triplet $\left(A_{l}, B_{l}, C_{l}\right)$ is minimal iff the matrices $C_{n_{j} ; j}$ among the matrices generalizing the norming constants to multipole situations (i.e., to $n_{j} \geq 2$ ) are invertible.

Example 6.3 (Double pole soliton solutions) In the Cases 2, 3 and 4 we plot the three distinct elements of the complex symmetric matrix $Q(x, t)$ for the same matrix triplet $\left(A_{r}, B_{r}, C_{r}\right)$, where

$$
\begin{gathered}
A_{r}=\left(\begin{array}{cccc}
3 & 0 & -1 & 0 \\
0 & 3 & 0 & -1 \\
0 & 0 & 3 & 0 \\
0 & 0 & 0 & 3
\end{array}\right), \\
C_{r}=-i\left(\begin{array}{cccc}
i & 2 i & 1 & 0 \\
2 i & 3 i & 0 & 1
\end{array}\right), \quad B_{r}=\left(\begin{array}{ll}
1 & 0 \\
0 & 1 \\
1 & 0 \\
0 & 1
\end{array}\right) .
\end{gathered}
$$

The plots show that in Case 3 (see figure 5) the elements of $Q(x, t)$ have the same finite singularity and that in Case 4 (see figure 6) these elements do not have any finite singularities. Moreover, the plots of Case 2 and 4 are very similar because of the "structures" of these two cases are very similar. Also for the double poles solutions we have not found necessary and sufficient conditions on the discrete eigenvalue and the two associated norming constants in order that, in Case 3 or in Case 4, there are no finite singularities. However, we observe that in the Case 4 

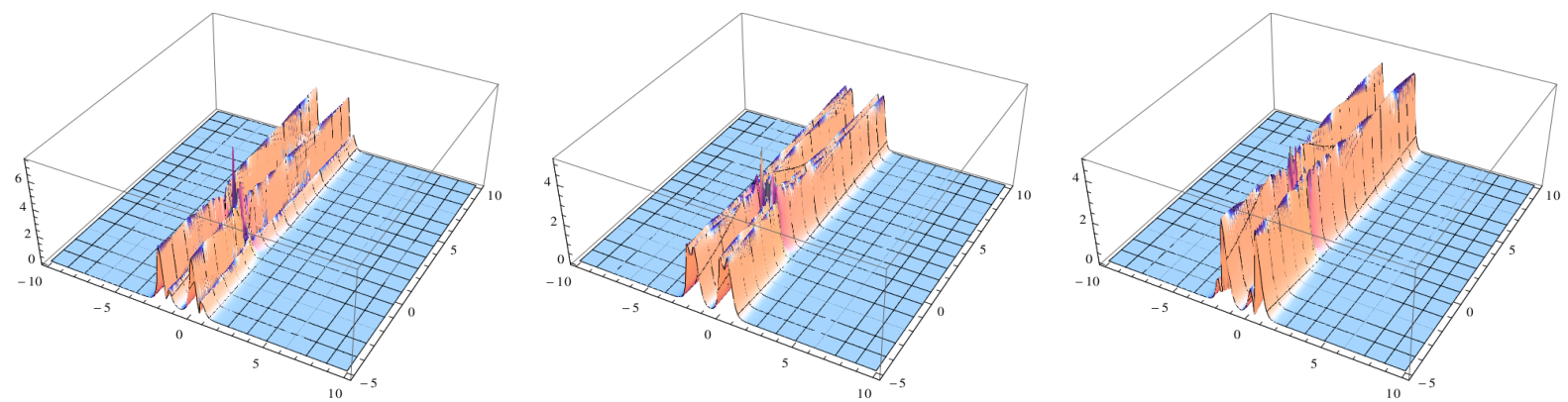

Figure 4: An example of a double pole solution in the focsing case.
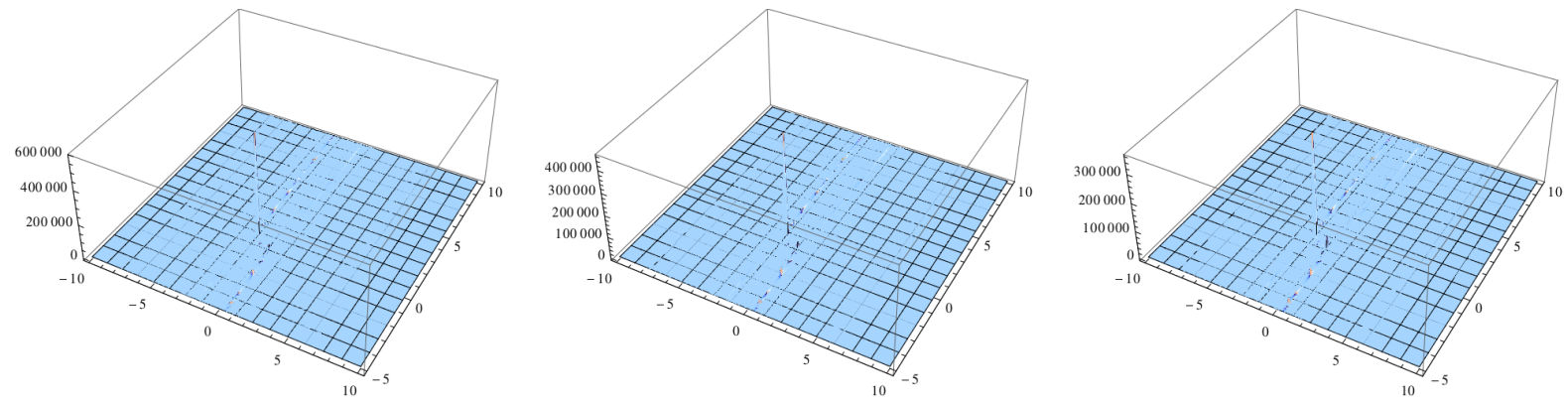

Figure 5: An example of a double pole solution in case 3. Note the presence of a finite singularity.
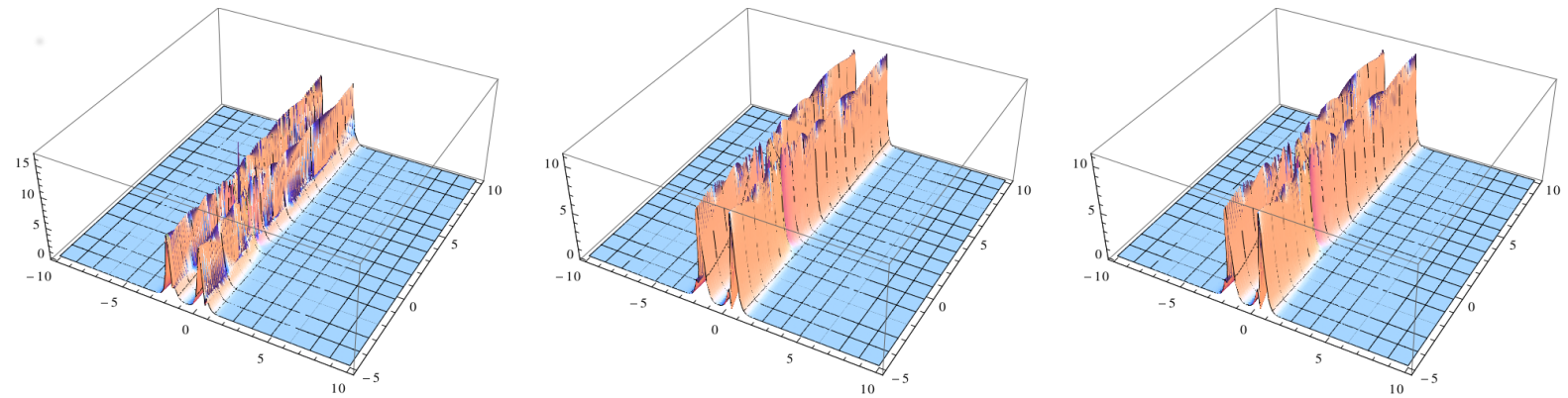

Figure 6: An example of a a double pole solution in case 4 . 


\section{Acknowledgments}

The research leading to this article has been partially supported by the Regione Autonoma Sardegna in the framework of the research programs IntegroDifferential equations and non-local problems and Algorithms and Models for Imaging Science [AMIS], and by INdAM-GNFM (Istituto Nazionale di Alta Matematica, National Institute of Advanced Mathematics - Gruppo Nazionale per la Fisica Matematica, National Group for Mathematical Physics).

\section{A Integral equations for kernel functions}

In this appendix we list the integral equations for the auxiliary functions $\bar{K}(x, y, t), K(x, y, t), L(x, y, t)$, and $\bar{L}(x, y, t)$. Their proof can be found in the literature $[2,6,24]$. For the sake of brevity, we omit the time variable in all equations.

We have the following Volterra integral equations for the kernel functions:

$$
\begin{aligned}
& \bar{K}^{\mathrm{up}}(x, y)=-\int_{x}^{\infty} d z Q(z) \bar{K}^{\mathrm{dn}}(z, z+y-x), \\
& \bar{K}^{\mathrm{dn}}(x, y)=-\frac{1}{2} R\left(\frac{1}{2}[x+y]\right)-\int_{x}^{\frac{1}{2}[x+y]} d z R(z) \bar{K}^{\mathrm{up}}(z, x+y-z), \\
& K^{\mathrm{up}}(x, y)=-\frac{1}{2} Q\left(\frac{1}{2}[x+y]\right)-\int_{x}^{\frac{1}{2}[x+y]} d z Q(z) K^{\mathrm{dn}}(z, x+y-z), \\
& K^{\mathrm{dn}}(x, y)=-\int_{x}^{\infty} d z R(z) K^{\mathrm{up}}(z, z+y-x),
\end{aligned}
$$


as well as

$$
\begin{aligned}
& L^{\mathrm{up}}(x, y)=\int_{-\infty}^{x} d z Q(z) L^{\mathrm{dn}}(z, z+y-x), \\
& L^{\mathrm{dn}}(x, y)=\frac{1}{2} R\left(\frac{1}{2}[x+y]\right)+\int_{x}^{\frac{1}{2}[x+y]} d z R(z) L^{\mathrm{up}}(z, x+y-z), \\
& \bar{L}^{\mathrm{up}}(x, y)=\frac{1}{2} Q\left(\frac{1}{2}[x+y]\right)+\int_{x}^{\frac{1}{2}[x+y]} d z Q(z) \bar{L}^{\mathrm{dn}}(z, x+y-z), \\
& \bar{L}^{\mathrm{dn}}(x, y)=\int_{-\infty}^{x} d z R(z) \bar{L}^{\mathrm{up}}(z, z+y-x) .
\end{aligned}
$$

Equations (A.1) and (A.2) obviously imply (2.8).

Suppose that $F(y)$ is a matrix function of $y \geq x \geq x_{0}$ or a matrix function of $y \leq x \leq x_{0}$. Then we define

$$
\mu(F, x)= \begin{cases}\int_{x}^{\infty} d y\|F(y)\|, & x \geq x_{0}, \\ \int_{-\infty}^{x} d y\|F(y)\|, & x \leq x_{0} .\end{cases}
$$

Then (A.1) and (A.2) imply

$$
\begin{aligned}
& \mu\left(\bar{K}^{\mathrm{up}}, x\right) \leq \int_{x}^{\infty} d z\|Q(z)\| \mu\left(\bar{K}^{\mathrm{dn}}, z\right), \\
& \mu\left(\bar{K}^{\mathrm{dn}}, x\right) \leq \int_{x}^{\infty} d z\|R(z)\|+\int_{x}^{\infty} d z\|R(z)\| \mu\left(\bar{K}^{\mathrm{up}}, z\right), \\
& \mu\left(\bar{K}^{\mathrm{up}}, x\right) \leq \int_{x}^{\infty} d z\|Q(z)\|+\int_{x}^{\infty} d z\|Q(z)\| \mu\left(K^{\mathrm{dn}}, z\right), \\
& \mu\left(\bar{K}^{\mathrm{dn}}, x\right) \leq \int_{x}^{\infty} d z\|R(z)\| \mu\left(K^{\mathrm{up}}, z\right),
\end{aligned}
$$

as well as

$$
\begin{aligned}
& \mu\left(L^{\mathrm{up}}, x\right) \leq \int_{-\infty}^{x} d z\|Q(z)\| \mu\left(L^{\mathrm{dn}}, x\right), \\
& \mu\left(L^{\mathrm{dn}}, x\right) \leq \int_{-\infty}^{x} d z\|R(z)\|+\int_{-\infty}^{x} d z\|R(z)\| \mu\left(L^{\mathrm{up}}, x\right), \\
& \mu\left(\bar{L}^{\mathrm{up}}, x\right) \leq \int_{-\infty}^{x} d z\|Q(z)\|+\int_{-\infty}^{x} d z\|Q(z)\| \mu\left(\bar{L}^{\mathrm{dn}}, z\right), \\
& \mu\left(\bar{L}^{\mathrm{dn}}, x\right) \leq \int_{-\infty}^{x} d z\|R(z)\| \mu\left(\bar{L}^{\mathrm{up}}, z\right) .
\end{aligned}
$$


The unique solvability of (A.1) and (A.2) now follows immediately by applying Gronwall's inequality.

Using Gronwall's inequality we get

$$
\begin{gathered}
\mu\left(\bar{K}^{\mathrm{up}}, x\right)+\mu\left(\bar{K}^{\mathrm{dn}}, x\right) \leq\left(\int_{x}^{\infty} d z\|Q(z)\|\right) \exp \left(\int_{x}^{\infty} d z\|Q(z)\|\right), \\
\mu\left(K^{\mathrm{up}}, x\right)+\mu\left(K^{\mathrm{dn}}, x\right) \leq\left(\int_{x}^{\infty} d z\|Q(z)\|\right) \exp \left(\int_{x}^{\infty} d z\|Q(z)\|\right), \\
\mu\left(L^{\mathrm{up}}, x\right)+\mu\left(L^{\mathrm{dn}}, x\right) \leq\left(\int_{-\infty}^{x} d z\|Q(z)\|\right) \exp \left(\int_{-\infty}^{x} d z\|Q(z)\|\right), \\
\mu\left(\bar{L}^{\mathrm{up}}, x\right)+\mu\left(\bar{L}^{\mathrm{dn}}, x\right) \leq\left(\int_{-\infty}^{x} d z\|Q(z)\|\right) \exp \left(\int_{-\infty}^{x} d z\|Q(z)\|\right),
\end{gathered}
$$

where we have used that $\|Q(z)\| \equiv\|R(z)\|$.

Equations (2.8a) and (2.8b) are to be interpreted as follows: The integral terms in (A.1) and (A.2) are continuous in $y \in[x,+\infty)$ and in $y \in(-\infty, x]$, respectively. As a result,

$$
\begin{gathered}
\lim _{z \rightarrow x^{+}}\left\|2 \bar{K}^{\mathrm{dn}}(x, 2 z-x)+R(z)\right\|=0, \\
\lim _{z \rightarrow x^{+}}\left\|2 K^{\mathrm{up}}(x, 2 z-x)+Q(z)\right\|=0, \\
\lim _{z \rightarrow x^{-}}\left\|2 L^{\mathrm{dn}}(x, 2 z-x)-R(z)\right\|=0, \\
\lim _{z \rightarrow x^{-}}\left\|2 \bar{L}^{\mathrm{up}}(x, 2 z-x)-Q(z)\right\|=0,
\end{gathered}
$$

where we have substituted $z=\frac{1}{2}[x+y]$. If the entries of $Q(x)$ and $Q_{x}$ both belong to $L^{1}(\mathbb{R}, d x)$, then $(2.8 \mathrm{a})$ and $(2.8 \mathrm{~b})$ are valid pointwise.

\section{References}

[1] M.J. Ablowitz, D.J. Kaup, A.C. Newell, and H. Segur, The inverse scattering transform - Fourier analysis for nonlinear problems, Stud. Appl. Math. 53, 249-315 (1974).

[2] M.J. Ablowitz, B. Prinari, and A.D. Trubatch, Discrete and Continuous Nonlinear Schrödinger Systems, Cambridge University Press, Cambridge, 2004. 
[3] T. Aktosun, Th. Busse, F. Demontis, and C. van der Mee, Symmetries for exact solutions to the nonlinear Schrödinger equation, Journal of Physics A 43, 025202 (2010).

[4] T. Aktosun, F. Demontis, and C. van der Mee, Exact solutions to the focusing nonlinear Schrödinger equation, Inverse Problems 23, 21712195 (2007).

[5] H. Bart, I. Gohberg, and M.A. Kaashoek, Minimal Factorization of Matrix and Operator Functions, Birkhäuser OT 1, Basel, 1979.

[6] F. Demontis, Direct and Inverse Scattering of the Matrix ZakharovShabat System, Ph.D. thesis, University of Cagliari, 2007; also: Lambert Acad. Publ., Saarbrücken, 2012.

[7] F. Demontis, S. Lombardo, M. Sommacal, F. Vargiu, Effective generation of closed-form soliton solutions of the continuous classical Heisenberg ferromagnet equation, Communications in Nonlinear Science and Numerical Simulation 64, 35-65 (2018).

[8] F. Demontis, G. Ortenzi, C. van der Mee, Exact Solutions of the Hirota Equation and Vortex Filaments Motion, Physica D 313, 61-80 (2015).

[9] F. Demontis, B. Prinari, C. van der Mee, and F. Vitale, The inverse scattering transform for the focusing nonlinear Schrödinger equation with asymmetric boundary conditions, J. Math. Phys. 55, 101505 (2014), 40 pp.

[10] F. Demontis and C. van der Mee, Marchenko equations and norming constants of the matrix Zakharov-Shabat system, Operators and Matrices 2, 79-113 (2008).

[11] F. Demontis and C. van der Mee, Explicit solutions of the cubic matrix nonlinear Schrödinger equation, Inverse Problems 24, 025020 (2008).

[12] K. Eckert, Ł. Zawitkowski, M.J. Leskinen, A. Sanpera, and M. Lewenstein, Ultracold atomic Bose and Fermi spinor gases in optical lattices, New Journal of Physics 9(5), 133 (2007), 27 pp. 
[13] L.D. Faddeev and L.D. Takhtajan, Hamiltonian Methods in the Theory of Solitons, Springer, Berlin and Heidelberg, 2007.

[14] G.H. Golub and C.F. Van Loan, Matrix Computations, John Hopkins Univ. Press, fourth ed., Baltimore, 2013.

[15] M. Grabowski, Second harmonic generation in periodically modulated media, Photonic Band Gaps and Localization, NATO ASI Series B, Vol. 308, 453-458, Springer US, Boston, 1993.

[16] M. Grabowski, Bichromatic wave propagation in periodically poled media, Phys. Rev. A 48, 2370-2373 (1993).

[17] Tin-Lun Ho and Sungkit Yip, Pairing of fermions with arbitrary spin, Phys. Rev. Lett. 82, 247-250 (1999).

[18] J-ichi Ieda, T. Miyakawa, and M. Wadati, Exact analysis of soliton dynamics in spinor Bose-einstein condensates, Phys. Rev. Lett. 93, 194102 (2004).

[19] J-ichi Ieda, M. Uchiyama, and M. Wadati, Dark soliton in $F=1$ spinor Bose-Einstein condensates, J. Phys. Soc. Jpn 75, 064002 (2006).

[20] P.B. Lunquist, D.R. Andersen, and Y.S. Kivshar, Multicolor solitons due to four wave mixing, Phys. Rev. E 57, 3551-3555 (1998).

[21] B. Prinari, F. Demontis, S. Li, T. Horikis, Inverse scattering transform and soliton solutions for square matrix nonlinear Schrödinger equations with non-zero boundary conditions, Physica D 368, 22-49, (2018).

[22] B. Prinari, A. Ortiz, C. van der Mee, and M. Grabowski, Inverse scattering transform and solitons for square matrix nonlinear matrix nonlinear Schrödinger equations, Stud. Appl. Math. 141, 308-352 (2018).

[23] M. Uchiyama, J-ichi Ieda, and M. Wadati, Inverse scattering method for square matrix nonlinear Schroedinger equation under nonvanishing boundary conditions, J. Math. Phys. 48, 013507 (2007). 
[24] C. van der Mee, Nonlinear Evolution Models of Integrable Type, SIMAI e-Lecture Notes 11, Torino, 2013.

[25] C. Wu, Competing orders in one dimensional spin-3/2 fermionic systems, Phys. Rev. Lett. 95, 266404 (2005).

[26] C. Wu, Hidden symmetry and quantum phases in spin-3/2 cold atomic systems, Modern Phys. Lett. B 20, 1707-1738 (2006). 